\title{
High-angular resolution observations towards OMC-2 FIR 4: Dissecting an intermediate-mass protocluster ${ }^{\star}$
}

\author{
A. López-Sepulcre ${ }^{1}$, V. Taquet ${ }^{1}$, Á. Sánchez-Monge² ${ }^{2}$ C. Ceccarelli ${ }^{1}$, C. Dominik ${ }^{3,4}$, M. Kama ${ }^{3}$, E. Caux ${ }^{5,6}$, \\ F. Fontani ${ }^{2}$, A. Fuente ${ }^{7}$, P. T. P. Ho ${ }^{8,9}$, R. Neri ${ }^{10}$, and Y. Shimajiri ${ }^{11}$ \\ ${ }^{1}$ UJF - Grenoble 1/CNRS-INSU, Institut de Planétologie et d'Astrophysique de Grenoble (IPAG) UMR 5274, 38041 Grenoble, \\ France \\ e-mail: ana.sepulcre@obs.ujf-grenoble.fr \\ 2 Osservatorio Astrofisico di Arcetri, Largo E. Fermi 5, 50124 Firenze, Italy \\ 3 Astronomical Institute Anton Pannekoek, University of Amsterdam, PO BOX 942491090 GE Amsterdam, The Netherlands \\ 4 Department of Astrophysics/IMAPP, Radboud University Nijmegen, 6525 AJ Nijmegen, The Netherlands \\ 5 Université de Toulouse, UPS-OMP, IRAP, 31028 Toulouse Cedex 4, France \\ ${ }^{6}$ CNRS, IRAP, 9 Av. Colonel Roche, BP 44346, 31028 Toulouse Cedex 4, France \\ 7 Observatorio Astronómico Nacional, PO Box 112, 28803 Alcalá de Henares, Madrid, Spain \\ 8 Institute of Astronomy and Astrophysics, Academia Sinica, PO Box 23-141, 106 Taipei, Taiwan \\ 9 Harvard-Smithsonian Center for Astrophysics, 60 Garden Street, Cambridge, MA 02138, USA \\ 10 IRAM, 300 rue de la piscine, 38406 Saint-Martin d'Hères, France \\ 11 Nobeyama Radio Observatory, 462-2 Nobeyama, Minamimaki, Minamisaku, 384-1305 Nagano, Japan
}

Received 13 December 2012 / Accepted 16 April 2013

\section{ABSTRACT}

\begin{abstract}
Context. Intermediate-mass stars are an important ingredient of our Galaxy and a key to understanding how high- and low-mass stars form in clusters. One of the closest known young intermediate-mass protoclusters is OMC-2 FIR 4, which is located at a distance of $420 \mathrm{pc}$ in Orion. This region is one of the few where the complete 500-2000 GHz spectrum has been observed with the heterodyne spectrometer HIFI on board the Herschel satellite, and unbiased spectral surveys at $0.8,1,2$, and $3 \mathrm{~mm}$ have been obtained with the JCMT and IRAM 30-m telescopes.

Aims. We aim to disentangle the core multiplicity, to investigate the morphology of this region in order to study the formation of a low- and intermediate-mass protostar cluster, and to aid in interpretation of the single-dish line profiles already in our hands.

Methods. We used the IRAM Plateau de Bure Interferometer to image OMC-2 FIR 4 in the 2-mm continuum emission, as well as in $\mathrm{DCO}^{+}(2-1), \mathrm{DCN}(2-1), \mathrm{C}^{34} \mathrm{~S}(3-2)$, and several $\mathrm{CH}_{3} \mathrm{OH}$ lines. In addition, we analysed observations of the $\mathrm{NH}_{3}(1,1)$ and $(2,2)$ inversion transitions that used the Very Large Array of the NRAO. The resulting maps have an angular resolution that allows us to resolve structures of $5^{\prime \prime}$, which is equivalent to $2000 \mathrm{AU}$.

Results. Our observations reveal three spatially resolved sources within OMC-2 FIR 4, of one or several solar masses each, with hints of further unresolved substructure within them. Two of these sources have elongated shapes and are associated with dust continuum emission peaks, thus likely containing at least one molecular core each. One of them also displays radio continuum emission, which may be attributed to a young B3-B4 star that dominates the overall luminosity output of the region. The third identified source displays a $\mathrm{DCO}^{+}(2-1)$ emission peak and weak dust continuum emission. Its higher abundance of $\mathrm{DCO}^{+}$relative to the other two regions suggests a lower temperature, hence its possible association with either a younger low-mass protostar or a starless core. It may alternatively be part of the colder envelope of OMC-2 FIR 4.

Conclusions. Our interferometric observations show the complexity of the intermediate-mass protocluster OMC-2 FIR 4, where multiple cores, chemical differentiation, and an ionised region all coexist within an area of only $10000 \mathrm{AU}$.
\end{abstract}

Key words. ISM: individual objects: OMC-2 FIR 4 - ISM: molecules

\section{Introduction}

Star formation starts in dense fragments within clouds of molecular gas and dust and ends when nuclear hydrogen burning begins at the centre of a newly formed star. During this evolution, the physical processes and different phases involved are not necessarily the same for stars of all masses. Indeed, in the field of star formation, there has traditionally been a division between low-mass stars $\left(M \lesssim 2 M_{\odot}\right)$, intermediate-mass (IM) stars $\left(2 M_{\odot}<M \lesssim 8 M_{\odot}\right)$, and high-mass stars $\left(M>8 M_{\odot}\right)$.

* Based on observations carried out with the IRAM Plateau de Bure Interferometer. IRAM is supported by the INSU/CNRS (France), MPG (Germany), and IGN (Spain).
In the past decades, most of the attention has been devoted to the study of low-mass and high-mass star formation, revealing differences between the two that are not only dynamical (Beuther et al. 2007; Zinnecker \& Yorke 2007), but also chemical in nature (Bottinelli 2006; Ceccarelli et al. 2007). The formation of IM stars represents the bridge between low-mass and high-mass star formation and can bring important pieces of information to understanding how differently the process of star formation works in the two mass extremes. As their high-mass counterparts, IM stars emit a non-negligible amount of radiation in the far ultraviolet (FUV), and are thus able to photodissociate and ionise their surroundings. Therefore, the study of IM star formation can aid the current debate on the formation 
Table 1. Parameters of our continuum observations and those of Shimajiri et al. (2008).

\begin{tabular}{lcccc}
\hline \hline Parameter & \multicolumn{2}{c}{ This work } & \multicolumn{2}{c}{ Shimajiri et al. (2008) } \\
\hline Interferometer & PdBI & PdBI & NMA & NMA \\
Frequency $(\mathrm{GHz})$ & 143.4 & 143.4 & 92 & 92 \\
Weighting & natural & uniform & natural & uniform \\
Primary beam $\left({ }^{\prime \prime}\right)$ & 35 & 35 & 82 & 82 \\
Baseline range $(\mathrm{k} \lambda)$ & $10-80$ & $10-80$ & $2.9-115$ & $2.9-115$ \\
Beam size $\left({ }^{\prime \prime}\right)$ & $4.87 \times 2.73$ & $3.74 \times 2.74$ & $6.96 \times 4.03$ & $6.55 \times 3.34$ \\
PA of the beam $\left(^{\circ}\right)$ & -0.76 & -12.98 & -34.59 & -40.31 \\
rms $\left(\mathrm{mJy} \mathrm{beam}^{-1}\right)$ & 4.9 & 4.6 & 1.2 & 1.4 \\
\hline
\end{tabular}

mechanism of high-mass stars (see Zinnecker \& Yorke 2007, for a review).

While low-mass stars may form in isolation or in loose stellar aggregates, high-mass stars are always seen to be formed in densely clustered environments. IM star formation also occurs in clustered mode (e.g. Fuente et al. 2007) and has been found to be the transition between loose low-mass aggregations and tight high-mass clusters (Testi et al. 1999). IM protoclusters represent excellent laboratories for studying clustered star formation at almost the full range of stellar masses. Observationally, they can typically be found closer $(d \lesssim 1 \mathrm{kpc}$ ) than high-mass protoclusters, and they are also less complex.

Despite all this, relatively little work has focussed on the early stages of IM star formation. Only a few individual sources have been studied in detail (e.g. Fuente et al. 2009; Crimier et al. 2009, 2010; Sánchez-Monge et al. 2010; Palau et al. 2011; van Kempen et al. 2012), and therefore much remains unknown about the formation and first evolutionary stages of these objects.

The present work focusses on the IM protocluster OMC2 FIR 4, which has a luminosity that has been reported to be between $50 L_{\odot}$ (Adams et al. 2012) and $1000 L_{\odot}$ (Crimier et al. 2009). It is located in the Orion A complex, 2 pc north of the famous Orion Nebula (M42) and the Trapezium OB association, and lies $\sim 20$ pc away from the Sun (Menten et al. 2007; Kim et al. 2008).

Thanks to its relative proximity, multiple studies have been carried out towards this region and the OMC-2/3 filament where it is located. These include continuum observations at several wavelengths (e.g. Chini et al. 1997; Johnstone \& Bally 1999; Nielbock et al. 2003) and various molecular line studies (e.g. Tatematsu 1993; Cesaroni \& Wilson 1994; Takahashi et al. 2008; Tatematsu et al. 2008; Liu et al. 2011; Li et al. 2012). Most of them, however, are coarse angular-resolution observations, which are very useful for investigating the overall structure of the whole filament, but not sufficient to resolve the substructure within the OMC-2 FIR 4 region. The only dedicated interferometric observations towards this source are Very Large Array (VLA) maps carried out at $3.6 \mathrm{~cm}$ by Reipurth et al. (1999) and a recent study conducted with the Nobeyama Millimeter Array (NMA) at $3 \mathrm{~mm}$ by Shimajiri et al. (2008; hereafter S08). The latter reveal several cores and the authors speculate that an external outflow driven by the nearby FIR 3 is responsible for triggering fragmentation in FIR 4, highlighting the complexity of this region.

OMC-2 FIR 4 is also one of the targets of the Herschel ${ }^{1}$ guaranteed time key programme Chemical HErschel Surveys of Star

\footnotetext{
1 Herschel is a European Space Agency (ESA) space observatory with science instruments provided by European-led principal investigator consortia and with important participation from the National Aeronautics and Space Administration (NASA).
}

forming regions $\left(\mathrm{CHESS}^{2}\right.$; Ceccarelli et al. 2010; Kama et al. 2012), which has conducted unbiased spectral surveys with the HIFI spectrometer (de Graauw et al. 2010) of eight star-forming regions, each representative of a particular aspect of the star formation process: evolutionary stage, mass of the forming star, or interaction with the surroundings. The HIFI half-power beam width ranges between $11^{\prime \prime}$ at $1900 \mathrm{GHz}$ and $41^{\prime \prime}$ at $500 \mathrm{GHz}$, thus covering most, if not all, the area of OMC-2 FIR 4. Highangular resolution observations are therefore a crucial tool for disentangling the information hidden in the HIFI line profiles.

With this in mind, we report 2-mm continuum and line observations performed with the IRAM Plateau de Bure Interferometer (PdBI) and ammonia maps obtained with the VLA towards OMC-2 FIR 4. We describe these observations in Sect. 2; in Sect. 3 we present the maps and spectra obtained, which we discuss and interpret in Sect. 4. Finally, Sect. 5 summarises the main conclusions of this work.

\section{Observations}

\subsection{Plateau de Bure observations}

We observed OMC-2 FIR 4 with the IRAM Plateau de Bure Interferometer (PdBI) in two tracks on 15 October 2010 and 15 April 2011, using the most compact D and C configurations of the array. The data presented in this paper cover a spectral window of $1.8 \mathrm{GHz}$ obtained with the WIDEX correlator, whose channel spacing is $1.95 \mathrm{MHz}\left(\sim 4 \mathrm{~km} \mathrm{~s}^{-1}\right)$, centred on a frequency of $143.4 \mathrm{GHz}$. The phase centre of the observations is $\alpha(\mathrm{J} 2000)=05^{\mathrm{h}} 35^{\mathrm{m}} 26.971^{\mathrm{s}}, \delta(\mathrm{J} 2000)=-05^{\circ} 09^{\prime} 56.77^{\prime \prime}$. The systemic velocity of OMC-2 FIR 4 is $V_{\mathrm{LSR}}=11.4 \mathrm{~km} \mathrm{~s}^{-1}$.

The bandpass of the receivers was calibrated by observing 0923+392 in Oct. 2010 and NRAO150 in Apr. 2011 with flux densities of $3.55 \mathrm{Jy}$ and $2.53 \mathrm{Jy}$, respectively. Amplitude and phase calibrations were achieved by monitoring $0430+052$ and $0528+134$, whose flux densities were determined to be 1.32 and $1.67 \mathrm{Jy}$, respectively, at $143.4 \mathrm{GHz}$. The uncertainty in the amplitude calibration is estimated to be $30 \%$.

The data were calibrated and analysed with the GILDAS ${ }^{3}$ software package developed at IRAM and the Observatoire de Grenoble. We obtained continuum maps from the line-free channels and subtracted the continuum from the line emission directly in the $(u, v)$-domain. The resulting $1 \sigma$ rms and beam size for the naturally weighted continuum map are $4.9 \mathrm{mJy}^{\mathrm{beam}}{ }^{-1}$ and 4 ". $87 \times 2$ '. 73 , respectively (see Table 1 ). The maps presented in this paper are not corrected for the primary beam, but we did apply the correction to measure the reported fluxes.

\footnotetext{
2 http://www-laog.obs.ujf-grenoble.fr/heberges/chess/ 3 The GILDAS package is available at http://www.iram.fr/ IRAMFR/GILDAS
} 


\subsection{Very Large Array ammonia observations}

The Very Large Array $\left(\mathrm{VLA}^{4}\right)$ was used to observe the ammonia $(J, K)=(1,1)$ and $(2,2)$ inversion transitions simultaneously (project AC556). Twenty adjacent pointing centres, covering OMC-2 and OMC-3 regions, were carried out on 29 July 2000 and 24 September 2000, with the array in the D (compact) configuration. The FWHM of the primary beam at the frequency of the observations is approximately $2^{\prime}$, and the field centres were separated by $1^{\prime}$ to ensure full sampling. Each field was observed for a total on-source integration time of approximately $30 \mathrm{~min}$. The absolute flux scale was set by observing the quasar $1328+307$ (3C 286), for which we adopted a flux of $2.5 \mathrm{Jy}$. The quasars 0605-085 and 0539-057 with bootstrapped fluxes of $2.4 \mathrm{Jy}$ and $1.0 \mathrm{Jy}$, respectively, were observed regularly to calibrate the gains and phases. Bandpass calibration was performed by observing the bright quasar $0316+413$ (3C84) with a flux density of 14.2 Jy (for July) and 17.5 Jy (for September).

The data reduction followed the VLA standard guidelines for calibration of high-frequency data, using the NRAO package AIPS. The $\mathrm{NH}_{3}(1,1)$ and $\mathrm{NH}_{3}(2,2)$ lines were observed simultaneously in the four IF correlator modes of the VLA (with two polarisations for each line), providing 63 channels with a spacing of $0.62 \mathrm{~km} \mathrm{~s}^{-1}$ across a bandwidth of $3.125 \mathrm{MHz}$, plus an additional continuum channel containing the central 75 per cent of the total bandwidth. The bandwidth was centred at the systemic velocity $V_{\mathrm{LSR}}=11.4 \mathrm{~km} \mathrm{~s}^{-1}$.

Calibrated uv-data were loaded in the software MIRIAD (Sault et al. 1995) to perform the imaging. While the continuum emission was found to be negligible (we measured an upper limit of about $2 \mathrm{mJy}$ at the frequency of the observations), we subtracted any possible related contribution from line-free channels using the task UVLIN. We combined and imaged the data from the central part of the mosaic, covering the OMC-2 region, with the task MOSSDI. The rms noise in the naturally weighted maps is $5 \mathrm{mJy}^{\text {beam }}{ }^{-1}$ per $0.62 \mathrm{~km} \mathrm{~s}^{-1}$ channel. We applied a $u v$-taper function of $60 \mathrm{k} \lambda\left(3^{\prime \prime}\right.$ in the image plane) to improve the signal-to-noise ratio $(\mathrm{S} / \mathrm{N})$. The resulting synthesised beam is $6^{\prime \prime} .2 \times 5^{\prime \prime} .3$, with $\mathrm{PA}=-5^{\circ}$.

\section{Results}

\subsection{Millimetre continuum emission}

Figure 1 shows our PdBI naturally and uniformly weighted 2-mm continuum maps. For comparison, these are overlaid on the 3-mm continuum images obtained by S08 with the Nobeyama Millimeter Array (NMA). From their uniformly weighted image, S08 identify 11 cores at $3 \mathrm{~mm}$ within OMC2 FIR 4, whose positions according to their Table 4 are marked in the figure. The parameters of our continuum observations and those of S08 are listed in Table 1.

The PdBI images allow us to resolve two elongated structures in OMC-2 FIR 4, separated by $\sim 5^{\prime \prime}$, surrounded by more extended emission that covers a total size of about $15^{\prime \prime}$. The uniform map suggests the presence of additional fragmentation that cannot be completely resolved at the angular resolution of the observations. On the other hand, the NMA images display fainter, more complex, and more extended emission, which is

\footnotetext{
4 The Very Large Array (VLA) is operated by the National Radio Astronomy Observatory (NRAO), a facility of the National Science Foundation operated under cooperative agreement by Associated Universities, Inc.
}

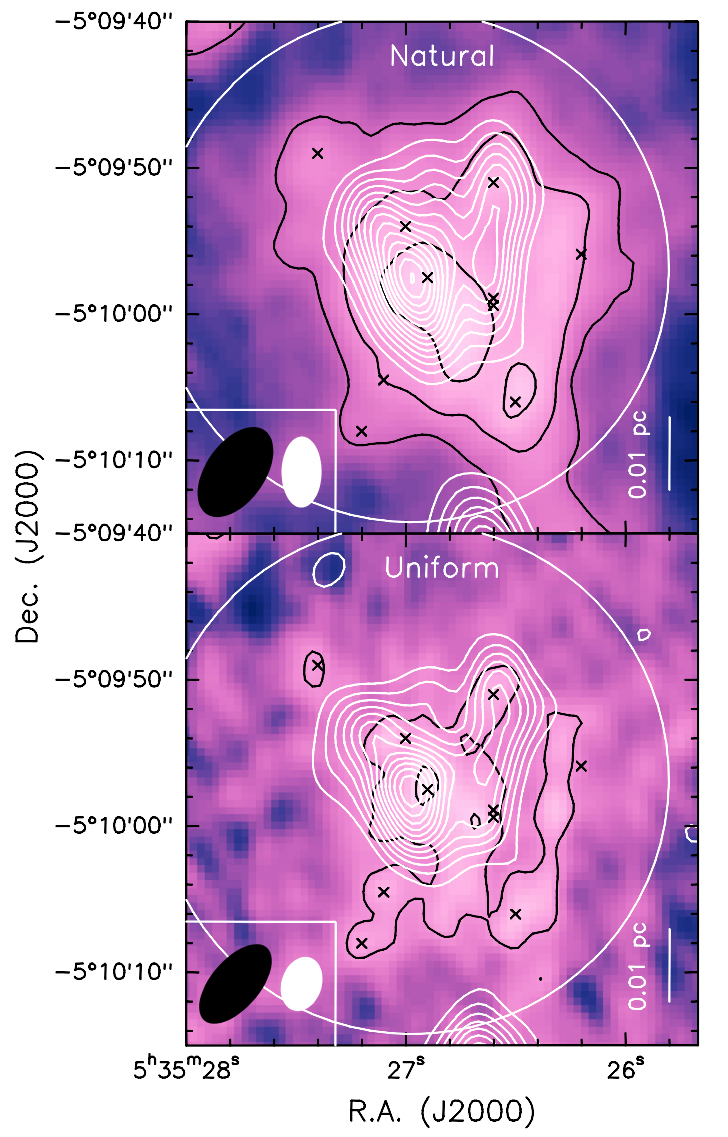

Fig. 1. Interferometric continuum maps of OMC-2 FIR 4 with natural (top) and uniform weighting (bottom). White contours represent the 2-mm continuum emission obtained with the PdBI, while black contours, as well as the background colour scale, correspond to the 3-mm continuum emission seen with the NMA (S08). Contours for both the PdBI and NMA maps start at $3 \sigma$ and increase by steps of $3 \sigma$. The bottom-left ellipses represent the respective beam sizes. The white open circles mark the primary beam FWHM for the PdBI observations. The black crosses mark the NMA 3-mm cores as reported in Table 4 of S08.

largely below the $3 \sigma$ level (first contour) in the case of the uniform map.

It is worth mentioning that the primary beam size of our PdBI observations is $35^{\prime \prime}$, so considerably smaller than that of the NMA maps, which is $82^{\prime \prime}$. Similarly, the shortest baseline attained with the NMA is $2.9 \mathrm{k} \lambda$ while that of the PdBI is $10 \mathrm{k} \lambda$. This results in a higher sensitivity of the NMA to extended structures. More specifically, our shortest baseline implies that our observations are sensitive to structures smaller than $17^{\prime \prime}$ down to a level of $10 \%$ (see Eq. (A.8) in Wilner \& Welch 1994; or Eq. (A.6) in Palau et al. 2010), while for the NMA images, this number is as high as $56^{\prime \prime}$. Thus, we are not sensitive to the cores identified by S08 that fall outside the outermost PdBI contour. In spite of this, we still have several concerns regarding the core identification made by S08. First, it was based on the uniform map, which has a low $\mathrm{S} / \mathrm{N}$; second, the coordinates given for some of the cores do not coincide with the corresponding emission peak positions in the image; and third, two pairs of cores lie within a beam size of each other. We believe the NMA natural map is more reliable than the uniform one given its higher sensitivity. This already shows a number of clearly separated cores, some of which appear to be followed also by the PdBI contours. In conclusion, even though we prefer to be cautious about the reality of the 11 cores reported in S08, we are convinced 
Table 2. Lines observed with the PdBI and VLA interferometers.

\begin{tabular}{|c|c|c|c|c|}
\hline Line & $\begin{array}{c}v^{a} \\
(\mathrm{GHz})\end{array}$ & $\begin{array}{l}E_{\text {up }} \\
(\mathrm{K})\end{array}$ & $\begin{array}{c}A_{\mathrm{ul}} \\
\left(\mathrm{s}^{-1}\right)\end{array}$ & $\begin{array}{c}1 \sigma \mathrm{rms}^{b} \\
\left(\mathrm{mJy} \mathrm{beam}^{-1}\right)\end{array}$ \\
\hline $\mathrm{CH}_{3} \mathrm{OH}\left(3_{1,3}-2_{1,2} \mathrm{~A}^{+}\right)$ & 143.865801 & 28.3 & $1.07 \times 10^{-5}$ & 3.7 \\
\hline $\mathrm{CH}_{3} \mathrm{OH}\left(3_{0,3}-2_{0,2} \mathrm{E}\right)$ & 145.093707 & 27.1 & $1.23 \times 10^{-5}$ & - \\
\hline $\mathrm{CH}_{3} \mathrm{OH}\left(3_{-1,3}-2_{-1,2} \mathrm{E}\right)$ & 145.097370 & 19.5 & $1.10 \times 10^{-5}$ & - \\
\hline $\mathrm{CH}_{3} \mathrm{OH}\left(3_{0,3}-2_{0,2} \mathrm{~A}^{+}\right)$ & 145.103152 & 13.9 & $1.23 \times 10^{-5}$ & - \\
\hline $\mathrm{CH}_{3} \mathrm{OH}\left(3_{2,2}-2_{2,1} \mathrm{~A}^{-}\right)$ & 145.124410 & 51.6 & $6.89 \times 10^{-6}$ & - \\
\hline $\mathrm{CH}_{3} \mathrm{OH}\left(3_{2,1}-2_{2,0} \mathrm{E}\right)$ & 145.126190 & 36.2 & $6.77 \times 10^{-6}$ & - \\
\hline $\mathrm{CH}_{3} \mathrm{OH}\left(3_{-2,2}-2_{-2,1} \mathrm{E}\right)$ & 145.126392 & 39.8 & $6.85 \times 10^{-6}$ & - \\
\hline $\mathrm{CH}_{3} \mathrm{OH}\left(3_{1,2}-2_{1,1} \mathrm{E}\right)$ & 145.131855 & 35.0 & $1.12 \times 10^{-5}$ & - \\
\hline $\mathrm{CH}_{3} \mathrm{OH}\left(3_{2,1}-2_{2,0} \mathrm{~A}^{+}\right)$ & 145.133460 & 51.6 & $6.89 \times 10^{-6}$ & - \\
\hline $\mathrm{DCO}^{+}(2-1)^{c}$ & 144.077289 & 10.4 & $2.12 \times 10^{-4}$ & 3.6 \\
\hline $\mathrm{C}^{34} \mathrm{~S}(3-2)$ & 144.617101 & 13.9 & $5.78 \times 10^{-5}$ & 3.2 \\
\hline $\operatorname{DCN}(2-1)^{c}$ & 144.828002 & 10.4 & $1.27 \times 10^{-4}$ & 3.4 \\
\hline $\mathrm{NH}_{3}(1,1)$ & 23.69450 & 23.3 & $1.68 \times 10^{-7}$ & 5.0 \\
\hline $\mathrm{NH}_{3}(2,2)$ & 23.72263 & 64.4 & $2.24 \times 10^{-7}$ & 4.7 \\
\hline
\end{tabular}

Notes. ${ }^{(a)}$ Millimetre line frequencies from the CDMS catalogue: http://www. astro.uni-koeln.de/cdms/. ${ }^{(b)}$ Indicates no map is presented in this paper. ${ }^{(c)}$ Transition with 6 hyperfine components.

that OMC-2 FIR 4 is composed of several cores. Based on our PdBI maps, we hereafter refer to the dominant eastern region as the main source, and to the western one as west source.

The continuum fluxes, which are measured inside the $5 \sigma$ contour of the corresponding maps, are $1.06 \pm 0.32 \mathrm{Jy}$ and $1.10 \pm 0.33 \mathrm{Jy}$ for the natural and uniform maps, respectively. We can estimate the mass of the emitting clump by considering that all the flux is emitted by dust. This is a reasonable assumption, since Reipurth et al. (1999) reported a 3.6-cm flux of $0.64 \mathrm{mJy}$ for OMC-2 FIR 4 (see Sect. 4.3), and therefore the contribution of free-free emission at $2 \mathrm{~mm}$ is negligible, i.e. about three orders of magnitude below the total flux we measure. Assuming that the dust emission is optically thin, the mass, $M_{\text {dust }}$ is given by

$M_{\mathrm{dust}}=\frac{S_{v} d^{2}}{\kappa_{v} B_{v}\left(T_{\mathrm{d}}\right) R_{\mathrm{d}}}$

where $S_{\gamma}$ is the flux we meaure inside the $5 \sigma$ contour at $2 \mathrm{~mm}, d$ the distance to OMC-2 FIR 4, $T_{\mathrm{d}}$ the dust temperature (adopted to be $50 \mathrm{~K}$ from S08), $B_{v}\left(T_{\mathrm{d}}\right)$ the Planck black body function for a temperature $T_{\mathrm{d}}$, and $R_{\mathrm{d}}$ the dust-to-gas ratio, assumed equal to 0.01 . The equation $\kappa_{v}=\kappa_{0}\left(\frac{v}{v_{0}}\right)^{\beta}$ is the frequency-dependent dust mass opacity coefficient. Chini et al. (1997) derived $\beta=2$ from the spectral energy distribution (SED) fit towards OMC2 FIR 1 and FIR 2. Since FIR 4 is in the same molecular filament as FIR 1 and FIR 2, we have adopted $\beta=2$, and $\kappa_{0}=1 \mathrm{~cm}^{2} \mathrm{~g}^{-1}$ at $v_{0}=250 \mathrm{GHz}$ (Ossenkopf \& Henning 1994).

The resulting mass, from the naturally-weighted $2-\mathrm{mm}$ continuum map, is $9 \pm 3 M_{\odot}$. This should be considered as a lower limit to the total mass of OMC-2 FIR 4 (dense cores plus envelope), since a percentage of the total flux is expected to be filtered out by the interferometer. We can estimate the fraction of flux lost with the aid of the work published by Crimier et al. (2009), who modelled the SED function observed towards OMC-2 FIR 4 at infrared and sub-millimetre wavelengths. The flux they predict at $2 \mathrm{~mm}$ is $2 \mathrm{Jy}$, implying a flux loss of $45 \%$ in our PdBI continuum observations.

Crimier et al. (2009) have derived a mass of $30 M_{\odot}$ for the envelope of OMC-2 FIR 4, i.e. higher than our estimate by a factor 3 . While this difference may be partly due to the loss of flux in our interferometric maps, we note that a different choice

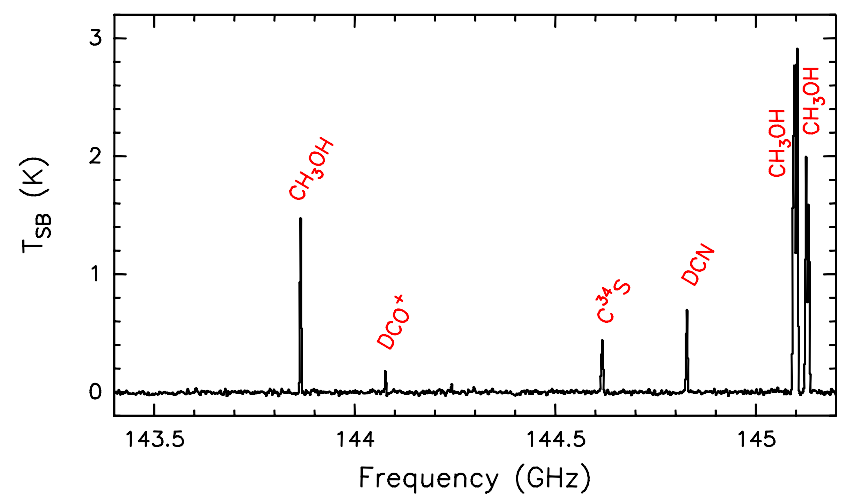

Fig. 2. PdBI spectrum obtained towards the phase centre of OMC2 FIR 4 with the WIDEX correlator. The species of the detected lines are labelled.

of dust temperature in Eq. (1) can significantly change the obtained mass. For example, adopting $T_{\mathrm{d}}=20 \mathrm{~K}$, which is a reasonable dust temperature for the source outer envelope (Lis et al. 1998; Jørgensen et al. 2006; Crimier et al. 2009), yields a mass of $26 M_{\odot}$.

\subsection{Line emission}

The list of detected transitions are presented in Table 2, together with the $1 \sigma$ rms values of the corresponding velocity channel maps (see Sects. 3.2.1 and 3.2.2). Out of the nine methanol lines detected, eight are blended due to the poor spectral resolution of the observations. Therefore, the methanol maps shown in this paper correspond to the only isolated transition we observe, i.e. $\mathrm{CH}_{3} \mathrm{OH}(3,1,+0-2,1,+0)$ at $143.87 \mathrm{GHz}$, whose upper level energy is $28 \mathrm{~K}$ and which is hereafter referred to as $\mathrm{CH}_{3} \mathrm{OH}$ for simplicity.

\subsubsection{PdBI molecular line spectra and maps}

The 1.8-GHz wide spectrum obtained at the peak coordinates of OMC-2 FIR 4 with the WIDEX correlator at PdBI is presented in Fig. 2, where the detected species are labelled. Since we are also in possession of IRAM 30-m single-pointing data towards 


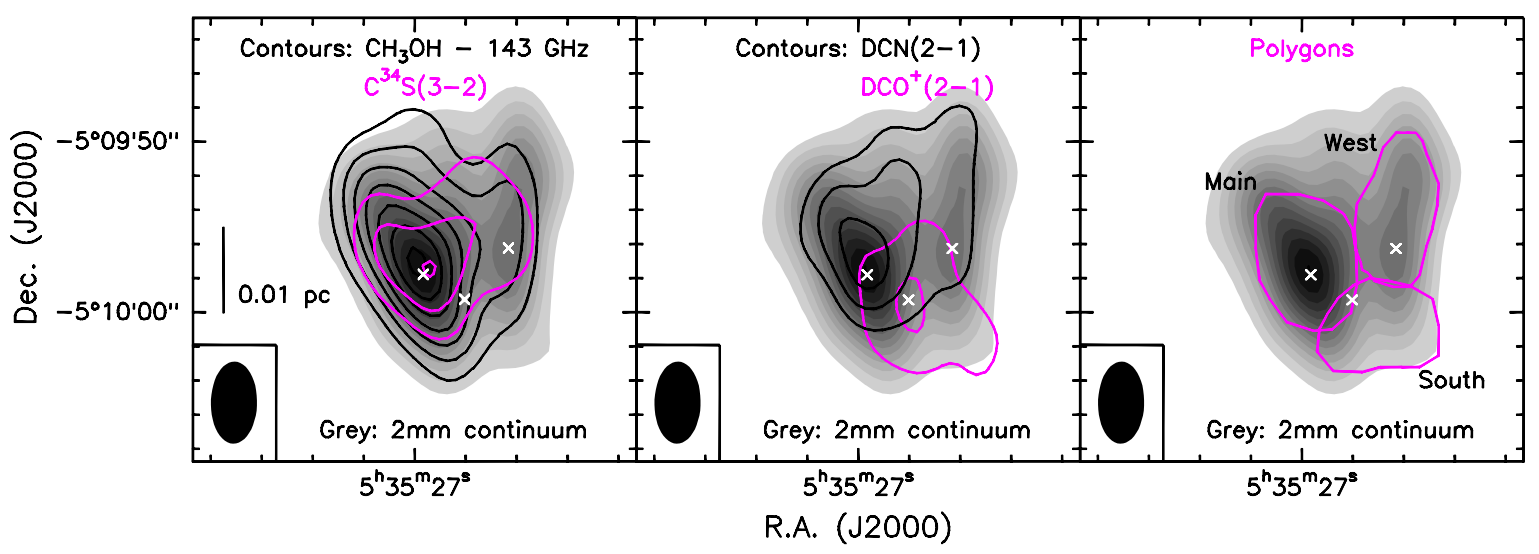

Fig. 3. Velocity-integrated PdBI contour maps obtained towards OMC-2 FIR 4, overlaid on the naturally weighted continuum map (grey scale). The bottom-left ellipse in each panel represents the beam size. Left: $\mathrm{CH}_{3} \mathrm{OH}(3,1,+0-2,1,+0)$ (black) and $\mathrm{C}^{34} \mathrm{~S}(3-2)$ (magenta). Centre: $\mathrm{DCN}(2-1)$ (black) and $\mathrm{DCO}^{+}(2-1)$ (magenta). All the contours start at $3 \sigma$ and increase by steps of $3 \sigma$. The $1 \sigma$ rms values are $0.16,0.14,0.17$, and $0.08 \mathrm{Jy}_{\text {beam }}^{-1} \mathrm{~km} \mathrm{~s}^{-1}$, respectively for the $\mathrm{CH}_{3} \mathrm{OH}, \mathrm{C}^{34} \mathrm{~S}(3-2), \mathrm{DCN}(2-1)$, and $\mathrm{DCO}^{+}(2-1)$ integrated maps. Right: Polygons representing the three differentiated components, main, west, and south (see text), used to extract average spectra. The white crosses mark the positions of the three sources identified in this work (see Table 4).

Table 3. Flux loss for the PdBI molecular lines.

\begin{tabular}{lc}
\hline \hline Line & $\begin{array}{c}\text { Flux loss } \\
(\%)\end{array}$ \\
\hline $\mathrm{CH}_{3} \mathrm{OH}$ & 13 \\
$\mathrm{DCO}^{+}(2-1)$ & 73 \\
$\mathrm{C}^{34} \mathrm{~S}(3-2)$ & 60 \\
$\mathrm{DCN}(2-1)$ & 72 \\
\hline
\end{tabular}

the same source (López-Sepulcre et al., in prep.), we provide in Table 3 an estimate of the velocity-integrated flux loss for each $\mathrm{PdBI}$ line. This ranges from $13 \%$ for $\mathrm{CH}_{3} \mathrm{OH}$ to $73 \%$ for $\mathrm{DCO}^{+}(2-1)$, and indicates the presence of an important contribution from extended emission in these tracers that is filtered out by the interferometer.

Given the low spectral resolution of the WIDEX spectrum (1.95 MHz, i.e. $4 \mathrm{~km} \mathrm{~s}^{-1}$ ), it is not possible to analyse the shape of the line profiles. However, this is beyond the scope of the present work, which primarily aims at investigating the spatial distribution of the emission in the different tracers. To this end we provide, for each millimetre molecular tracer, velocityintegrated maps in Fig. 3, and velocity channel maps in Fig. 4. The synthesised beam size of these images is $4 . .8 \times 2$.' 7 , and is depicted on the bottom left-hand corner of each panel in the figures.

If we focus first on the integrated maps (Fig. 3), the morphological complexity of the source becomes evident from a comparison of the emission distribution in the different tracers:

- The $\mathrm{CH}_{3} \mathrm{OH}$ emission is dominated by the main source (see Sect. 3.1 above), which displays an elongated shape, and shows hints of a secondary component towards the west (the above mentioned west source), mimicking the spatial distribution of the 2-mm continuum emission. This morphology does not change when seen in the blended $\mathrm{CH}_{3} \mathrm{OH}$ lines around $145 \mathrm{GHz}$.

- $C^{34} \mathrm{~S}(3-2)$ displays a more compact emission that traces both the main and west sources.

- The DCN(2-1) emission peaks at the main source, but it expands towards the west and the north, following the elongated morphology of the west source as seen in the continuum images.
- $\mathrm{DCO}^{+}(2-1)$ peaks to the south of the main and west sources, a different region that we name south source, which is not traced well by any other line.

In summary, from the integrated maps we conclude that each molecular line traces the region differently. We differentiate three spatially separated emission components, which we have named main, west, and south sources, lying $\sim 0.01 \mathrm{pc}$ (i.e. 2000 AU) apart from each other. These regions are schematically depicted in the right-hand panel of Fig. 3 by three magenta polygons that have been manually defined to pinpoint similar central areas for each while keeping approximately their morphological shapes. From these we have extracted the respective continuum and line fluxes for each tracer. The resulting fractional fluxes with respect to the total flux, which are measured inside the $3 \sigma$ map contour for the lines and the $5 \sigma$ contour for the continuum map, are listed in Table 4 . The central coordinates of each source correspond to the emission peaks of the 2-mm continuum image for the main and west sources, and those of the $\mathrm{DCO}^{+}(2-1)$ map for the south one. With the exception of $\mathrm{DCO}^{+}(2-1)$, the sum of fluxes is smaller than the total flux in the whole region owing to the relatively small area of each polygon. The sum amounts to $136 \%$ in the case of $\mathrm{DCO}^{+}(2-1)$ partly because the polygons corresponding to the main and south regions overlap around the position of the peak emission, and partly because there is an amount of contributing flux outside the $3 \sigma$ contour. This table reflects the predominance of the main source's flux over that of the other two for all the tracers except $\mathrm{DCO}^{+}(2-1)$, for which the south source dominates the overall emission.

Using Eq. (1), we have computed the mass, $M$, of each source for two values of the dust temperature: 20 and $50 \mathrm{~K}$. These are listed in Table 5, together with the corresponding source-averaged gas column densities derived from the equation:

$N_{\mathrm{H}_{2}}=\frac{M}{\mu m_{\mathrm{H}} \Omega d^{2}}$

where $m_{\mathrm{H}}$ is the mass of the hydrogen atom, $\mu=2.33$ is the mean molecular mass in units of hydrogen atom masses, and $\Omega$ the solid angle subtended by the dust continuum emission. Assuming the depth of the region equals the projected one (i.e. $5000 \mathrm{AU}$ ), we derive an average density in the range $0.9-2.6 \times 10^{7} \mathrm{~cm}^{-3}$ for OMC-2 FIR 4 . 
A\&A 556, A62 (2013)

Table 4. OMC-2 FIR 4 sources: coordinates and fractional fluxes for each tracer.

\begin{tabular}{lccccccc}
\hline \hline Source & $\mathrm{RA}(\mathrm{J} 2000)$ & Dec $(\mathrm{J} 2000)$ & $\mathrm{CH}_{3} \mathrm{OH}$ & $\mathrm{C}^{34} \mathrm{~S}(3-2)$ & $\mathrm{DCN}(2-1)$ & $\mathrm{DCO}^{+}(2-1)$ & Cont. (natural) \\
\hline Total $^{a}$ & & & 13.0 & 3.7 & 6.3 & 1.2 & 1.1 \\
\hline Main $(\%)$ & $05^{\mathrm{h}} 35^{\mathrm{m}} 26.97^{\mathrm{s}}$ & $-05^{\circ} 09^{\prime} 57.8^{\prime \prime}$ & 49 & 51 & 61 & 39 & 35 \\
West $(\%)$ & $05^{\mathrm{h}} 35^{\mathrm{m}} 26.63^{\mathrm{s}}$ & $-05^{\circ} 09^{\prime} 56.2^{\prime \prime}$ & 15 & 25 & 30 & 32 & 18 \\
South $(\%)$ & $05^{\mathrm{h}} 35^{\mathrm{m}} 26.80^{\mathrm{s}}$ & $-05^{\circ} 09^{\prime} 59.3^{\prime \prime}$ & 13 & 11 & 12 & 65 & 12 \\
\hline
\end{tabular}

Notes. ${ }^{(a)}$ Measured within the $5 \sigma$ and $3 \sigma$ contour for the continuum and line maps, respectively; flux units in Jy for continuum and Jy km s ${ }^{-1}$ for lines.

Table 5. OMC-2 FIR 4 sources: masses and column densities ${ }^{a}$.

\begin{tabular}{lcccc}
\hline \hline Source & $M(20 \mathrm{~K})$ & $M(50 \mathrm{~K})$ & $N_{\mathrm{H}_{2}}(20 \mathrm{~K})$ & $N_{\mathrm{H}_{2}}(50 \mathrm{~K})$ \\
& $\left(M_{\odot}\right)$ & $\left(M_{\odot}\right)$ & $\left(10^{23} \mathrm{~cm}^{-2}\right)$ & $\left(10^{23} \mathrm{~cm}^{-2}\right)$ \\
\hline Total & 25.7 & 9.2 & 19.2 & 6.9 \\
\hline Main & 9.1 & 3.2 & 29.0 & 10.4 \\
West & 4.7 & 1.7 & 18.7 & 6.7 \\
South & 3.0 & 1.1 & 12.4 & 4.4 \\
\hline
\end{tabular}

Notes. ${ }^{(a)}$ Derived from the naturally-weighted 2-mm continuum map.

The channel maps in Fig. 4 reveal more details about the spatial distribution of the molecular line emission. Despite the low spectral resolution of the observations, the emission in all the tracers but $\mathrm{DCO}^{+}(2-1)$ is detected above $5 \sigma$ across five velocity channels (i.e. covering a width of about $18 \mathrm{~km} \mathrm{~s}^{-1}$ ), and therefore some kinematical information can already be extracted from these maps. We note, however, that in the case of DCN(21) this velocity spread is partly due to the hyperfine splitting (see Sect. 3.2.3 below). In Fig. 5 we show the average spectra within the main, west, and south sources, defined by the polygons shown in Fig. 3 (right panel). Figure 6 presents the first moment maps for the four molecular tracers observed with the PdBI (colour scale), with overlaying contours of the corresponding velocity-integrated maps. The spectra, channel maps, and velocity maps reflect the following properties:

- The main source displays a velocity gradient in the southwest to north-east directions.

- The west source as seen by $\mathrm{CH}_{3} \mathrm{OH}$ and $\mathrm{C}^{34} \mathrm{~S}(3-2)$ appears slightly blue-shifted with respect to the systemic velocity of the source.

- The $\mathrm{DCO}^{+}(2-1)$ emission is significantly the narrowest in line width and concentrates almost exclusively on the south source.

- A secondary, well-separated condensation is distinguished in the $\mathrm{CH}_{3} \mathrm{OH}$ and DCN(2-1) maps to the south-west of the main source, suggesting the presence of an additional physical source. The $\mathrm{DCO}^{+}$emission also extends towards this area.

While the channel maps suggest the presence of more sources apart from the three we have identified so far, we cannot confirm it with $100 \%$ certainty at this spatial resolution, and even less guess how many more are present. We therefore conclude, as stated above, that there are at least three distinct sources within OMC-2 FIR 4, leaving open the possibility of further multiplicity within each of these three regions.

\subsubsection{Ammonia emission}

The $\mathrm{NH}_{3}(1,1)$ and $(2,2)$ line spectra seen towards the peak $\mathrm{NH}_{3}(1,1)$ emission coordinates are shown in Fig. 7, with the different hyperfine components marked. The limited band width of the correlator allows us to cover only one pair of satellites for each transition, apart form the main component. In the case of $\mathrm{NH}_{3}(2,2)$, only the main component is detected.

Velocity-integrated ammonia maps are shown in Fig. 8, where the emission corresponds to the main hyperfine component of the $\mathrm{NH}_{3}(1,1)$ and $\mathrm{NH}_{3}(2,2)$ transitions. These are overlaid on the 2-mm continuum PdBI image for comparison. We stress that the presence of extended emission that was filtered out by the VLA results in a relatively high $1 \sigma \mathrm{rms}$ level in the clean maps. Indeed, Li et al. (2012) have recently published these ammonia data merged with single-dish maps obtained with the Green Bank Telescope (GBT), and a comparison with our VLAonly spectra indicates a flux loss of about $40 \%$. Nevertheless, in terms of morphology, the $\mathrm{NH}_{3}$ emission is well defined around OMC-2 FIR 4 and peaks to the south of the main source. The emission appears extended, with a roundish shape, and covers an area which is larger than that of the 2-mm continuum.

For completeness, in Fig. 9 we present the channel maps for the two $\mathrm{NH}_{3}$ transitions, with the velocity of the channels given in the top right-hand corner of each panel in $\mathrm{km} \mathrm{s}^{-1}$. Unlike the millimetre molecular spectra (Sect. 3.2.1), the $\mathrm{NH}_{3}$ lines are rather narrow, as can be seen from the velocity range covered by the maps (see also the spectra in Fig. 7 and the FWHM values in Table 8). This may partly stem from the better spectral resolution and lower sensitivity of these observations. Indeed, the channel spacing of the VLA observations $\left(\sim 0.6 \mathrm{~km} \mathrm{~s}^{-1}\right)$ is substantially smaller than that of the WIDEX PdBI spectra.

The channel maps show extended emission around the central coordinates of OMC-2 FIR 4, and several other sources of emission: two towards the north-east and the south-west, where FIR 3 and FIR 5 are located, respectively, and a third one towards the south-east at the systemic velocity of the source (11.4 $\mathrm{km} \mathrm{s}^{-1}$ ), also seen in the single-dish 1.3-mm maps carried out by Chini et al. (1997).

\subsubsection{Derivation of physical parameters}

Both the $\mathrm{DCN}(2-1)$ and $\mathrm{DCO}^{+}(2-1)$ lines are split into six hyperfine components. For $\mathrm{DCO}^{+}(2-1)$, all of them lie within a velocity range of $0.3 \mathrm{~km} \mathrm{~s}^{-1}$, while for $\mathrm{DCN}(2-1)$ they span $8 \mathrm{~km} \mathrm{~s}^{-1}$. As a result, at the spectral resolution of our observations, they are all blended and unresolved, so a hyperfine analysis is not possible. We have thus performed a Gaussian fit to each line and adopted a range of $T_{\mathrm{ex}}$ between 10 and $100 \mathrm{~K}$ to estimate a range of column densities from

$N_{\mathrm{mol}}=\frac{8 \pi v^{3}}{c^{3}} \frac{Q}{A_{\mathrm{ul}} g_{\mathrm{u}}} \frac{e^{\frac{E_{\mathrm{u}}}{k T_{\mathrm{ex}}}}}{e^{\frac{h v}{k T_{\mathrm{ex}}}}-1} \frac{\int T_{\mathrm{L}} \mathrm{d} v}{J\left(T_{\mathrm{ex}}\right)-J\left(T_{\mathrm{bg}}\right)}$

where $v, c, h, k, A_{\mathrm{ul}}, \tau_{\text {peak }}, \Delta V, Q, g_{\mathrm{u}}, E_{\text {up }}$, and $\int T_{\mathrm{L}} \mathrm{d} v$ are, respectively, the frequency of the transition, the speed of light, 

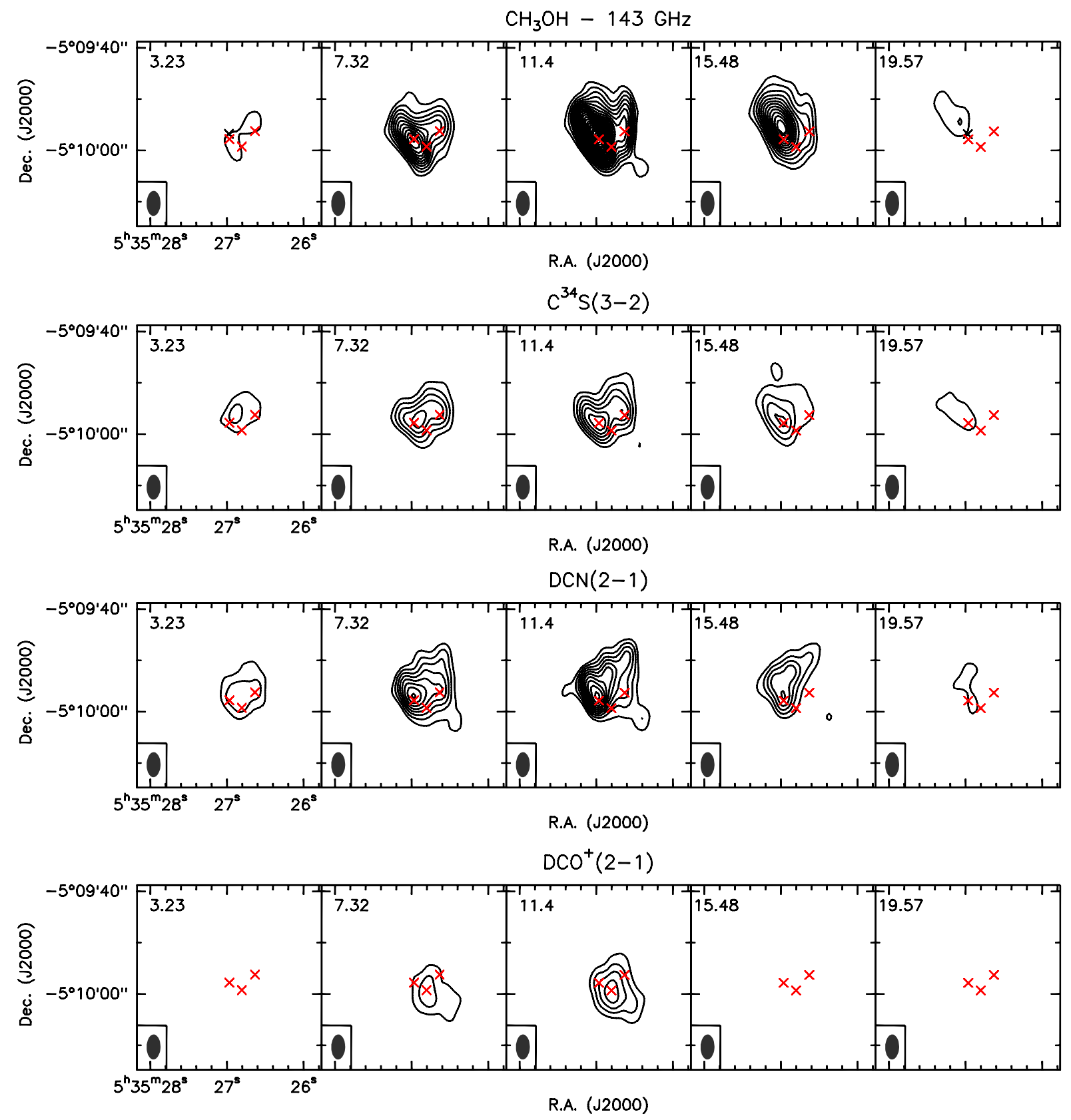

Fig. 4. Channel contour maps of each line detected with PdBI towards OMC-2 FIR 4. Contours start at $5 \sigma$ and increase by steps of $5 \sigma$. The $1 \sigma$ rms values are listed in Table 2. The velocity of each channel is marked on the top-left corner of each panel. Black crosses mark the phase centre of the observations, i.e. $\alpha(\mathrm{J} 2000)=05^{\mathrm{h}} 35^{\mathrm{m}} 26.971^{\mathrm{s}}, \delta(\mathrm{J} 2000)=-05^{\circ} 09^{\prime} 56.77^{\prime \prime}$, while red crosses mark the positions of the three sources identified in this work (see Table 4). The bottom-left ellipses in each panel represent the beam sizes.

Planck constant, Boltzmann constant, the spontaneous emission rate, the optical depth at the line intensity peak, the line FWHM, the partition function, the upper state degeneracy, the upper level energy, and the velocity-integrated line intensity. This equation assumes optically thin conditions, and $J(T)=\frac{h v / k}{e^{h v / k T}-1}$, with $T_{\text {bg }}=2.7 \mathrm{~K}$. We applied the same approach to the $\mathrm{C}^{34} \mathrm{~S}(3-2)$ transition.

The results of the Gaussian fits and the derived molecular column densities for each source are shown in Table 6, where it can be seen that we are indeed dealing with optically thin conditions. Some of the rows in this table probably correspond to "artificial" sources, since not all the sources we have identified are well-defined or traced by all the lines, as explained above. We nevertheless present them for comparison and completeness.
The abundances of each molecule in the different sources are also listed. They have been obtained from the ratio of $N_{\text {mol }}$ to the gas column density, $N$. For the latter, we have used the values listed in Table 5, adopting both $T_{\mathrm{d}}=20 \mathrm{~K}$ and $T_{\mathrm{d}}=50 \mathrm{~K}$.

As for the methanol lines, we analysed them by means of a large velocity gradient (LVG) code, originally developed by Ceccarelli et al. (2003) and modified to include the methanol collisional coefficients with $\mathrm{H}_{2}$ by Pottage et al. (2004). For this analysis, we used the methanol lines obtained by averaging the emission over the entire OMC-2 FIR 4 clump. The line intensities thus measured are listed in Table 7, where the values shown for the $145-\mathrm{GHz}$ lines do not correspond to single transitions but to ensembles of blended methanol lines around the indicated frequency and transition. We ran a large grid of models to cover 


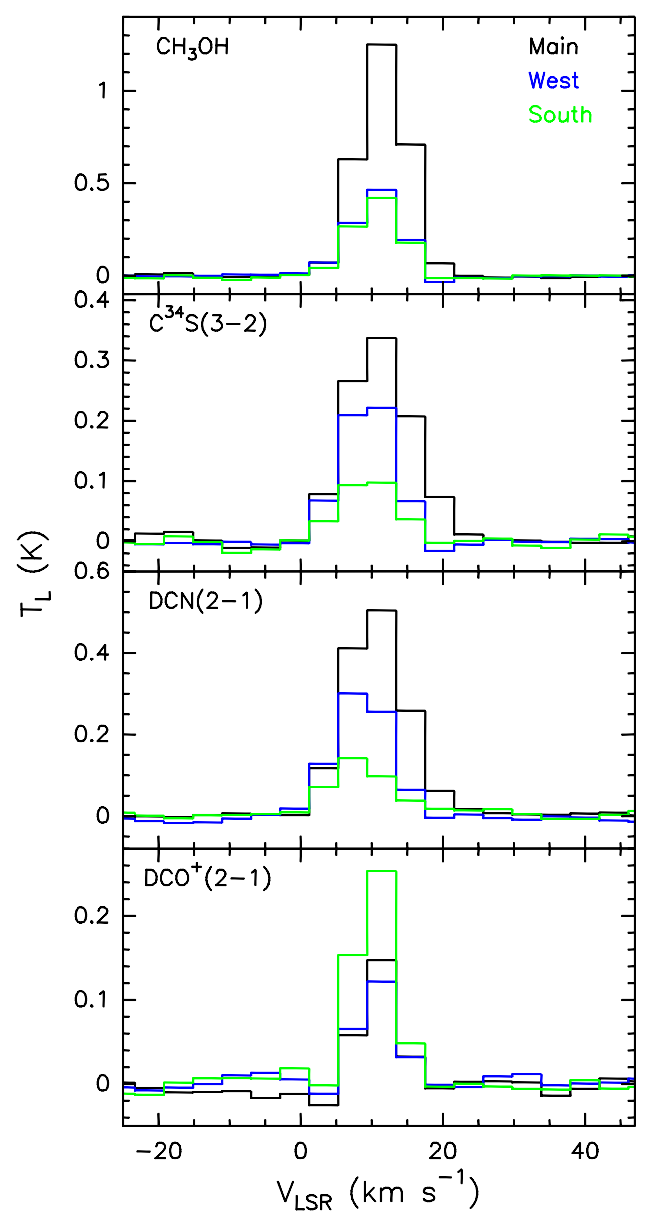

Fig. 5. Spectra in the three main sources identified in OMC-2 FIR 4 (see text) for each line tracer. The vertical scale is the brightness temperature of the line, $T_{\mathrm{L}}$, averaged across the area of the corresponding polygon (see Fig. 3).

a wide parameter space: $N\left(\mathrm{CH}_{3} \mathrm{OH}\right)$ from $10^{13}$ to $4 \times 10^{15} \mathrm{~cm}^{-2}$, density from $10^{4}$ to $10^{9} \mathrm{~cm}^{-3}$ and temperature from 10 to $200 \mathrm{~K}$. The observed lines were compared with the LVG model predictions and the best fit solution was found by minimising the $\chi^{2}$ value with respect to the three above parameters, assuming a source size of $15^{\prime \prime} \times 15^{\prime \prime}$, as measured in our maps for the whole OMC-2 FIR 4 clump. We note that for the blended lines the predicted intensities were obtained by adding the intensity by each single line in the velocity interval.

We found that the $\mathrm{CH}_{3} \mathrm{OH}$ column density is very well constrained between 0.6 and $1 \times 10^{15} \mathrm{~cm}^{-2}$, roughly consistent with the value of $\sim 2 \times 10^{14} \mathrm{~cm}^{-2}$ reported by Kama et al. (2010) from the early results of the HIFI data. The density is between 0.2 and $1 \times 10^{7} \mathrm{~cm}^{-3}$, a range of values that matches the average density we obtain from the millimetre continuum emission (see Sect. 3.2.1). The predicted optical depths of the $\mathrm{CH}_{3} \mathrm{OH}$ lines are low, around a few times $10^{-2}$.

The observed lines do not allow to constrain the temperature, except that it is higher than $20 \mathrm{~K}$. The higher the $\mathrm{CH}_{3} \mathrm{OH}$ column density, the higher the gas temperature. For example, if $N\left(\mathrm{CH}_{3} \mathrm{OH}\right)$ is $8 \times 10^{14} \mathrm{~cm}^{-2}$ then the gas temperature is higher than $50 \mathrm{~K}$; for $N\left(\mathrm{CH}_{3} \mathrm{OH}\right)=1 \times 10^{15} \mathrm{~cm}^{-2}$ the gas temperature is higher than $120 \mathrm{~K}$. More methanol transitions will be necessary to constrain the temperature better, but our analysis already shows that the gas emitting the methanol lines must be relatively dense. The best fit solutions of the LVG modelling have a reduced $\chi^{2}$ below 0.5 and are summarised in Table 7 .
Finally, we performed a hyperfine line fit to the two ammonia spectra in Fig. 7 using the standard $\mathrm{NH}_{3}(1,1)$ and $\mathrm{NH}_{3}(2,2)$ methods in CLASS. We subsequently derived the corresponding rotational and kinetic temperatures, as well as the $\mathrm{NH}_{3}$ column density, following the steps summarised in Busquet et al. (2009) and assuming a beam-filling factor equal to 1 . The results are reported in Table 8, where the peak synthesised beam temperature, $T_{\mathrm{SB}}$, the velocity, $V_{\mathrm{LSR}}$, and the optical depth, $\tau_{\text {main }}$ refer to the main component of the transition. We note that the errors on the temperature and column density estimates are close to or greater than the values themselves, mostly due to the large errors on $T_{\mathrm{SB}}$ and $\tau$. Using the $\mathrm{H}_{2}$ column densities listed in Table 5, we obtain an $\mathrm{NH}_{3}$ abundance that is slightly above $10^{-10}$. While this value is about two orders of magnitude less than what is typically reported in the literature, there are studies of ammonia in dense molecular cores that find values as low as ours or even lower (e.g. Foster et al. 2009; Wootten 1995).

\section{Discussion}

\subsection{Source multiplicity within OMC-2 FIR 4}

Several studies (Jørgensen et al. 2006, S08; Crimier et al. 2009; Kama et al. 2010) suggest that OMC-2 FIR 4 contains three main components:

\footnotetext{
- a large-scale cool envelope about 10000 AU (i.e. 0.05 pc) across,

- an inner hot core, with kinetic temperatures above $100 \mathrm{~K}$ and around $400 \mathrm{AU}$ in size,

- a shock region produced by the interaction of an external bipolar outflow driven by the nearby OMC-2 FIR 3, which lies to the north-east of FIR 4.
}

In addition, S08 report the presence of 11 cores seen at 3-mm inside this region, and speculate that such fragmentation has been externally triggered by the FIR 3 outflow.

The emission of all the maps presented in Sect. 3 covers roughly $10000 \mathrm{AU}(0.05 \mathrm{pc})$ and therefore traces the envelope component of OMC-2 FIR 4. In particular, this component appears to be better traced by the $\mathrm{NH}_{3}$ maps, which are essentially single-peaked and display the most extended emission, similar in size and morphology to the structures detected in previous single-dish observations (e.g. Chini et al. 1997; the ASTE maps of Shimajiri et al. 2008). The temperature derived for the ammonia emission, $\sim 30 \mathrm{~K}$, is typical of protostellar (in our case protocluster) envelopes (e.g. Olmi et al. 2012).

At the resolution of our PdBI observations we cannot confirm the existence of the 11 cores claimed by S08, despite its being slightly better than that of the NMA observations. Inspection of the different maps presented in this work allows us to clearly separate three regions, denoted by the identifiers main, west, and south (Sect. 3.2.1). It appears, however, that our 2-mm continuum and some molecular line maps indeed hint at the presence of multiple smaller condensations within the three regions we detect, but these will need to be confirmed by higher angular resolution observations.

At this point, it is worth mentioning that the hot-core hypothesis proposed by Crimier et al. (2009) is based on a single source at the centre of the large-scale envelope. S08 and our new observations show that the envelope component breaks down at scales of $2000 \mathrm{AU}$, so much larger than the presumed hot core size (with a radius of $440 \mathrm{AU}$ ). Only high-angular resolution imaging of typical hot core tracers (e.g. complex organic molecules) can observationally prove the existence of a hot core. 
A. López-Sepulcre et al.: Dissecting an intermediate-mass protocluster

Table 6. OMC-2 FIR 4 sources: Gaussian fit results, column densities and abundances for each PdBI tracer.

\begin{tabular}{|c|c|c|c|c|c|c|c|c|c|}
\hline Tracer & Source & $\begin{array}{r}T_{\mathrm{L}} \\
(\mathrm{K}) \\
\end{array}$ & $\begin{array}{c}V_{\mathrm{LSR}} \\
\left(\mathrm{km} \mathrm{s}^{-1}\right)\end{array}$ & $\begin{array}{l}F W H M \\
\left(\mathrm{~km} \mathrm{~s}^{-1}\right)\end{array}$ & $\begin{array}{c}T_{\mathrm{ex}^{a}} \\
(\mathrm{~K})\end{array}$ & $\tau$ & $\begin{array}{c}N_{\mathrm{mol}} \\
\left(10^{12} \mathrm{~cm}^{-2}\right)\end{array}$ & $\begin{array}{c}\text { Abu. }(20 \mathrm{~K})^{b} \\
\left(10^{-12}\right)\end{array}$ & $\begin{array}{c}\text { Abu. }(50 \mathrm{~K})^{b} \\
\left(10^{-12}\right)\end{array}$ \\
\hline \multirow{3}{*}{$C^{34} S(3-2)$} & Main & $0.37(0.01)$ & $10.9(0.1)$ & $11.3(0.2)$ & $10-100$ & $0.055-0.004$ & $15.3-38.2$ & $5.3-13.2$ & $14.7-36.7$ \\
\hline & West & $0.38(0.01)$ & $9.3(0.1)$ & $10.5(0.2)$ & $10-100$ & $0.040-0.003$ & $8.8-22.2$ & $4.7-11.9$ & $13.1-33.1$ \\
\hline & South & $0.15(0.01)$ & $9.3(0.2)$ & $10.7(0.6)$ & $10-100$ & $0.017-0.001$ & $4.0-10.3$ & $3.2-8.3$ & $9.1-23.4$ \\
\hline \multirow{3}{*}{$\operatorname{DCN}(2-1)$} & Main & $0.55(0.01)$ & $10.6(0.1)$ & $10.0(0.1)$ & $10-100$ & $0.085-0.006$ & $6.8-22.0$ & $2.3-7.6$ & $6.5-21.2$ \\
\hline & West & $0.67(0.01)$ & $9.8(0.1)$ & 9.8 & $10-100$ & $0.052-0.003$ & $3.7-12.5$ & 6.7 & 18.7 \\
\hline & South & $0.29(0.01)$ & $9.0(0.1)$ & $10.2(0.4)$ & $10-100$ & $0.022-0.001$ & $1.8-6.0$ & $1.5-4.8$ & $4.1-13.6$ \\
\hline \multirow{3}{*}{$\mathrm{DCO}^{+}(2-1)$} & Main & $0.19(0.01)$ & $10.8(0.2)$ & $6.1(0.3)$ & $10-100$ & $0.024-0.002$ & $0.6-2.3$ & $0.2-0.8$ & $0.6-2.2$ \\
\hline & West & $0.20(0.01)$ & $11.1(0.2)$ & $6.3(0.3)$ & $10-100$ & $0.020-0.001$ & $0.6-2.1$ & $0.3-1.1$ & $0.9-3.1$ \\
\hline & South & $0.38(0.01)$ & $10.3(0.1)$ & $6.3(0.2)$ & $10-100$ & $0.044-0.003$ & $1.3-4.5$ & $1.0-3.6$ & $3.0-10.2$ \\
\hline
\end{tabular}

Notes. ${ }^{(a)}$ Adopted values. ${ }^{(b)}$ Abundance determined using the dust temperature indicated in brackets to derive the total gas column density (see Table 5).

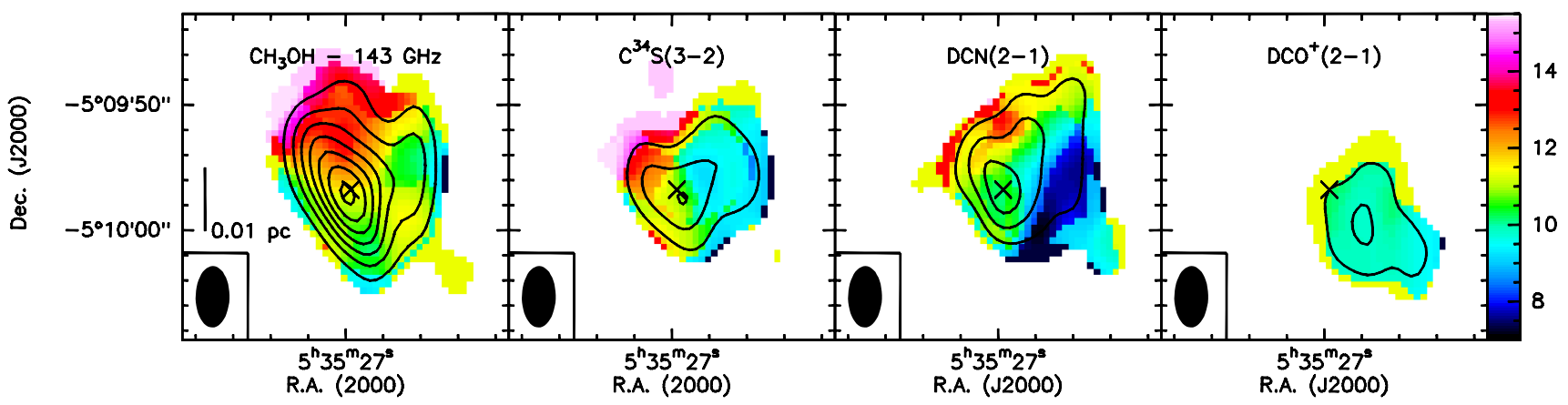

Fig. 6. Velocity maps (colour scale, in $\mathrm{km} \mathrm{s}^{-1}$ ) of each line detected with PdBI towards OMC-2 FIR 4, with overlaying contours of the corresponding velocity-integrated maps. Contour levels are as in Fig. 3. The bottom-left ellipse in each panel represents the beam size. Crosses mark the phase centre of the observations, i.e. $\alpha(\mathrm{J} 2000)=05^{\mathrm{h}} 35^{\mathrm{m}} 26.971^{\mathrm{s}}, \delta(\mathrm{J} 2000)=-05^{\circ} 09^{\prime} 56.77^{\prime \prime}$.

Table 7. $\mathrm{CH}_{3} \mathrm{OH}$ line intensities and results of the LVG analysis.

\begin{tabular}{cccc}
\hline \hline \multicolumn{4}{c}{$\mathrm{CH}_{3} \mathrm{OH}$ line intensities } \\
\hline Transition & $\begin{array}{c}v \\
\end{array}$ & $\int T_{\mathrm{SB}} \mathrm{d} V$ & Notes \\
& $(\mathrm{GHz})$ & $\left(\mathrm{K} \mathrm{km} \mathrm{s}^{-1}\right)$ & \\
\hline $3_{1,3}-2_{1,2} \mathrm{~A}^{+}$ & 143.866 & $5.2 \pm 1.6$ & isolated \\
$3_{-1,3}-2_{-1,2} \mathrm{E}^{+}$ & 145.097 & $28.8 \pm 8.7$ & 3 blended lines \\
$3_{-2,2}-2_{-2,1} \mathrm{E}^{+}$ & 145.126 & $13.9 \pm 4.2$ & 5 blended lines \\
\hline \multicolumn{4}{c}{ Results of the LVG analysis } \\
\hline $\mathrm{N}\left(\mathrm{CH}_{3} \mathrm{OH}\right)$ & Density & Temperature \\
$\left(10^{14} \mathrm{~cm}^{-2}\right)$ & $\left(10^{6} \mathrm{~cm}^{-3}\right)$ & $(\mathrm{K})$ & \\
\hline 6 & $>2$ & $20-50$ \\
8 & $>2$ & $>50$ & \\
10 & $2-10$ & $>120$ \\
\hline
\end{tabular}

As shown in Fig. 6, a velocity difference of about $6 \mathrm{~km} \mathrm{~s}^{-1}$ can be seen between the south-west and the north-east extremes of the main source in the $\mathrm{CH}_{3} \mathrm{OH}$ and $\mathrm{C}^{34} \mathrm{~S}(3-2)$ maps, with the north-eastern tip being the most red-shifted. S08 find the same behaviour in their single-dish methanol maps. Additional interferometric observations of $\mathrm{CO}$ and $\mathrm{SiO}$ lines provided the same authors with morphological and dynamical evidence that led them to suggest that there is an interaction between the northeastern edge of OMC-2 FIR 4 and the molecular outflow driven by the nearby FIR 3. The red-shifted emission we detect from $\mathrm{CH}_{3} \mathrm{OH}$ and $\mathrm{C}^{34} \mathrm{~S}(3-2)$ may also be explained by this scenario; i.e., it arises from shocked gas from the interaction between the FIR 3 outflow and the dense gas of FIR 4. It might alternatively be tracing the FIR 3 outflow itself, which cannot be detected much beyond the boundary of the FIR 4 clump due to the limited

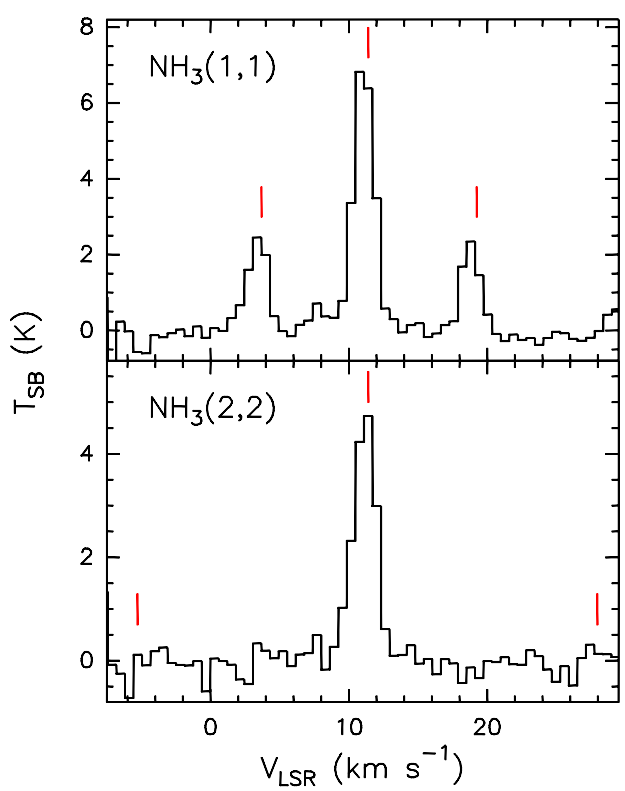

Fig. 7. VLA ammonia spectra obtained towards the peak coordinates of OMC-2 FIR 4, with a channel width of $0.62 \mathrm{~km} \mathrm{~s}^{-1}$. The different hyperfine components are marked with red vertical lines.

sensitivity of the PdBI to more extended structures. More observations are needed to confirm the external outflow interpretation, since with our data alone we are not able to conclude that this is indeed the case. Other possibilities exist that could cause a velocity gradient like the one we detect, such as rotation or internal outflowing/expanding motions. 
Table 8. Results of the line fitting to the $\mathrm{NH}_{3}(1,1)$ and $(2,2)$ lines and derived physical parameters.

\begin{tabular}{|c|c|c|c|c|c|}
\hline \multicolumn{6}{|c|}{ Results of the hyperfine line fitting } \\
\hline Line & $\begin{array}{l}T_{\mathrm{SB}} \\
(\mathrm{K})\end{array}$ & $\begin{array}{c}V_{\mathrm{LSR}} \\
\left(\mathrm{km} \mathrm{s}^{-1}\right)\end{array}$ & $\begin{array}{c}\Delta V \\
\left(\mathrm{~km} \mathrm{~s}^{-1}\right)\end{array}$ & $\tau_{\text {main }}$ & \\
\hline $\mathrm{NH}_{3}(1,1)$ & $7.5(2.7)$ & $11.1(0.1)$ & $1.5(0.1)$ & $0.7(0.2)$ & \\
\hline $\mathrm{NH}_{3}(2,2)$ & $4.9(9.8)$ & $11.2(0.1)$ & $1.9(0.1)$ & $0.1(0.2)$ & \\
\hline \multicolumn{6}{|c|}{ Physical parameters } \\
\hline $\begin{array}{l}T_{\mathrm{ex}} \\
(\mathrm{K})\end{array}$ & $\begin{array}{l}T_{\text {rot }} \\
(\mathrm{K})\end{array}$ & $\begin{array}{l}T_{\text {kin }} \\
(\mathrm{K})\end{array}$ & $\begin{array}{c}N_{\mathrm{NH}_{3}} \\
\left(10^{14} \mathrm{~cm}^{-2}\right)\end{array}$ & $\begin{array}{c}\text { Abu. }(20 \mathrm{~K})^{a} \\
\left(10^{-10}\right)\end{array}$ & $\begin{array}{c}\text { Abu. }(50 \mathrm{~K})^{a} \\
\left(10^{-10}\right)\end{array}$ \\
\hline 18.2 & 22.7 & 29.6 & 2.6 & 1.4 & 3.8 \\
\hline
\end{tabular}

Notes. ${ }^{(a)}$ Abundance determined using the dust temperature indicated in brackets to derive the total gas column density (see Table 5).

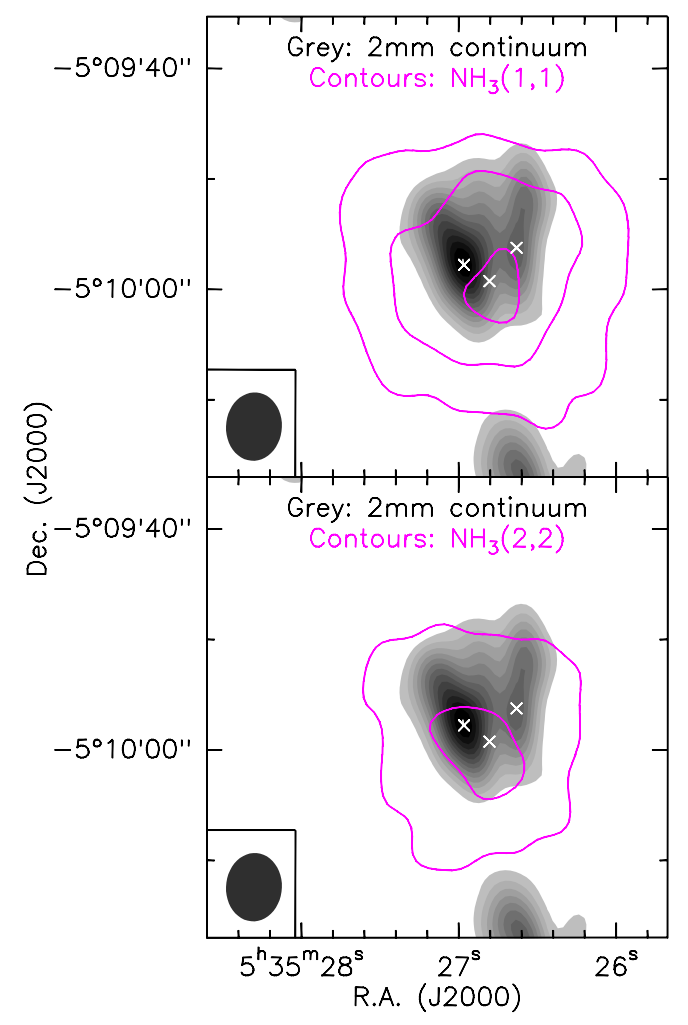

Fig. 8. Velocity-integrated VLA ammonia contour maps obtained towards OMC-2 FIR 4, overlaid on the naturally weighted $2 \mathrm{~mm}$ continuum map (grey scale). Contours for all the maps start at $3 \sigma$ and increase by steps of $3 \sigma$. The $1 \sigma \mathrm{rms}$ values are $20 \mathrm{mJy}$ beam ${ }^{-1} \mathrm{~km} \mathrm{~s}^{-1}$ for both $\mathrm{NH}_{3}(1,1)$ and $\mathrm{NH}_{3}(2,2)$. White crosses mark the positions of the three sources identified in this work (see Table 4).

\subsection{Nature of the sources}

Our observations allow us to distinguish three separate sources that may or may not present more substructure:

1. The main source, at the nominal coordinates and systemic velocity $\left(11.4 \mathrm{~km} \mathrm{~s}^{-1}\right)$ of OMC-2 FIR 4 . It is the strongest 2 -mm continuum and methanol source in the region. Its elongated shape suggests that it harbours two or more individual cores, although this hypothesis can only be confirmed with higher spatial resolution imaging. The red-shifted velocity of its northern portion might indicate an interaction with the red-shifted lobe of the external outflow driven by FIR 3.

2. The west source, with a systemic velocity of $9-10 \mathrm{~km} \mathrm{~s}^{-1}$. It is well traced by $\mathrm{C}^{34} \mathrm{~S}(3-2)$ and $\mathrm{DCN}(2-1)$, and weaker than the main source emission.
3. The south source, also with $V_{\mathrm{LSR}}=9-10 \mathrm{~km} \mathrm{~s}^{-1}$, best traced by $\mathrm{DCO}^{+}(2-1)$. It is the faintest methanol, $\mathrm{DCN}$, and 2-mm continuum source, and has no significant emission in $\mathrm{C}^{34} \mathrm{~S}(3-2)$. Its association with $\mathrm{DCO}^{+}$indicates this region is likely colder than the other two, suggesting that it contains either a starless core or a protostar in an earlier evolutionary stage or with a lower mass (e.g. Jørgensen et al. 2004). Alternatively, since it does not display any emission peak in the 2-mm continuum image, it may not be a separated molecular core, but part of the cold external envelope of OMC-2 FIR 4.

From the results of our LVG analysis on the $\mathrm{CH}_{3} \mathrm{OH}$ lines, we find that the entire OMC-2 FIR 4 clump is rather dense $(n>$ $2 \times 10^{6} \mathrm{~cm}^{-3}$ at $15^{\prime \prime}$, i.e. $6000 \mathrm{AU}$ ). This suggests that the three regions we identify in this work, which are all together smaller in size when compared to the whole emission considered in the $\mathrm{CH}_{3} \mathrm{OH}$ analysis, may be even denser. We note that the density we derive is in good agreement with S08 and with the predictions made by Crimier et al. (2009), both of which report a value of $10^{6} \mathrm{~cm}^{-3}$.

\subsection{An ionised region in OMC-2 FIR 4}

Radio-continuum observations of the OMC2/3 filament were carried out with the VLA by Reipurth et al. (1999) at $3.6 \mathrm{~cm}$. At the position of OMC-2 FIR 4, they detected an elongated source that the authors interpret as a radio jet. When comparing their map with our PdBI map, despite the different angular resolutions (i.e. $\sim 4^{\prime \prime}$ for the PdBI maps, $8^{\prime \prime}$ for the VLA maps), we find that the position and shape of the radio source coincides fairly well within the pointing errors of the two maps, with the emission distribution of our 2-mm continuum and methanol PdBI images, which are both elongated in the same direction as the VLA source (see Fig. 10).

While Reipurth et al. (1999) attribute the radio continuum emission to a jet, the interferometric observations performed towards this source so far (e.g. S08, this work) have failed to detect molecular line emission at the high velocities indicative of a jet or outflow emanating from this region. This leads us to consider a different possibility, namely that the radio emission arises from a very compact gas cavity ionised by a central young star, i.e. an HII region, which is either surrounded by a shell of dust and molecular gas best traced by the $2-\mathrm{mm}$ continuum and $\mathrm{CH}_{3} \mathrm{OH}$ emission, or else harbours two or more unresolved molecular dense cores within it. The HII region scenario is a plausible explanation for the continuum radio emission, since IM protostars can also produce sufficiently intense HII regions to be easily detected at centimetre wavelengths (e.g. Thompson 1984). 

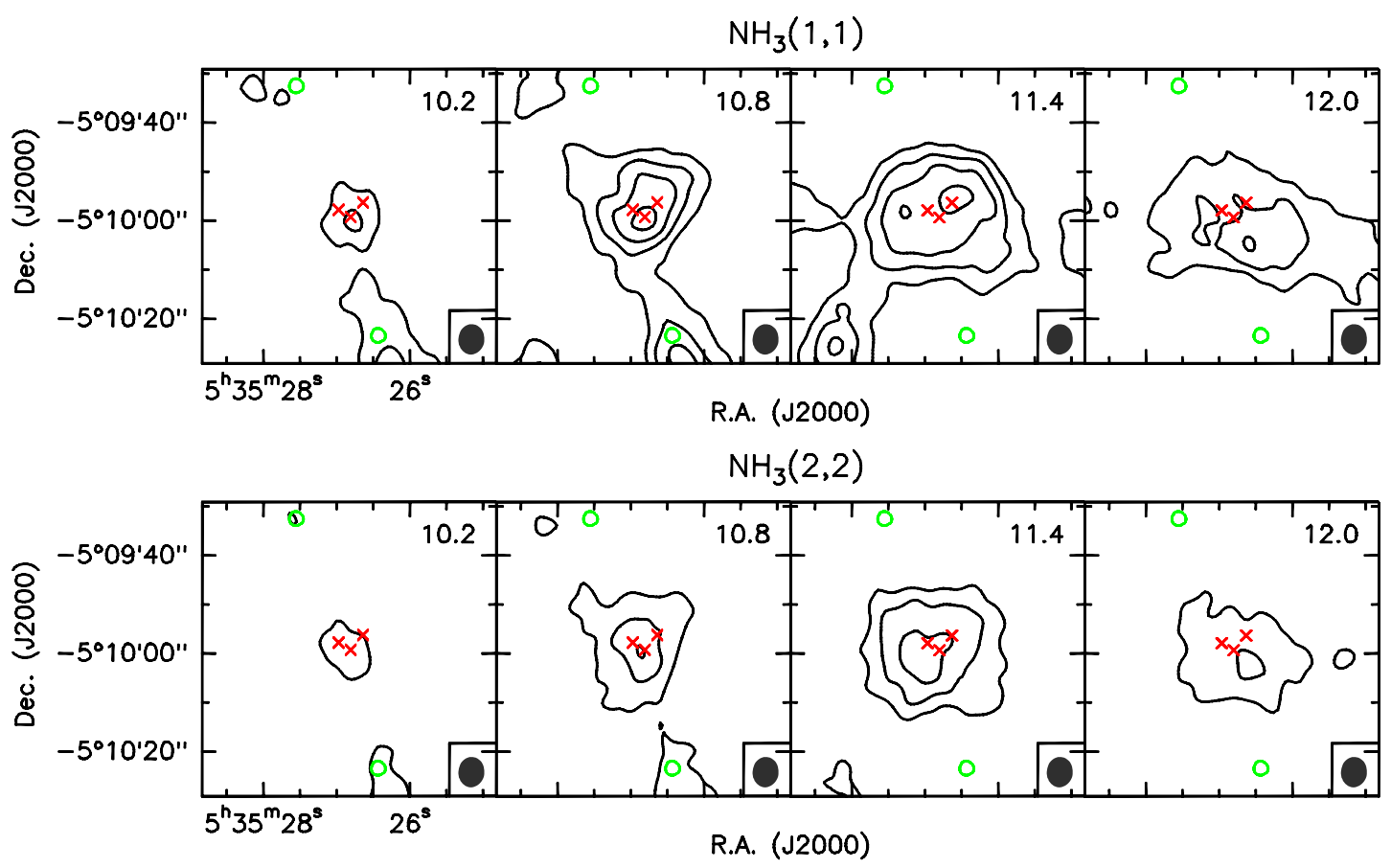

Fig. 9. VLA NH $3(1,1)$ and $\mathrm{NH}_{3}(2,2)$ channel maps towards OMC-2 FIR 4. Contours start at $5 \sigma$ and increase by steps of $5 \sigma$. The $1 \sigma$ rms values are $5.0 \mathrm{mJy}_{\text {beam }}^{-1}$ for $\mathrm{NH}_{3}(1,1)$ and $4.7 \mathrm{mJy}^{-1}$ beam ${ }^{-1}$ for $\mathrm{NH}_{3}(2,2)$. The velocity of each channel is marked in the top-right corner of each panel in units of $\mathrm{km} \mathrm{s}^{-1}$. Red crosses mark the positions of the three sources identified in this work (see Table 4). Green circles mark the position of FIR 3 (NE) and FIR 5 (SW) according to the $1.2 \mathrm{~mm}$ observations of Chini et al. (1997). The bottom-right ellipses in each panel represent the beam sizes.

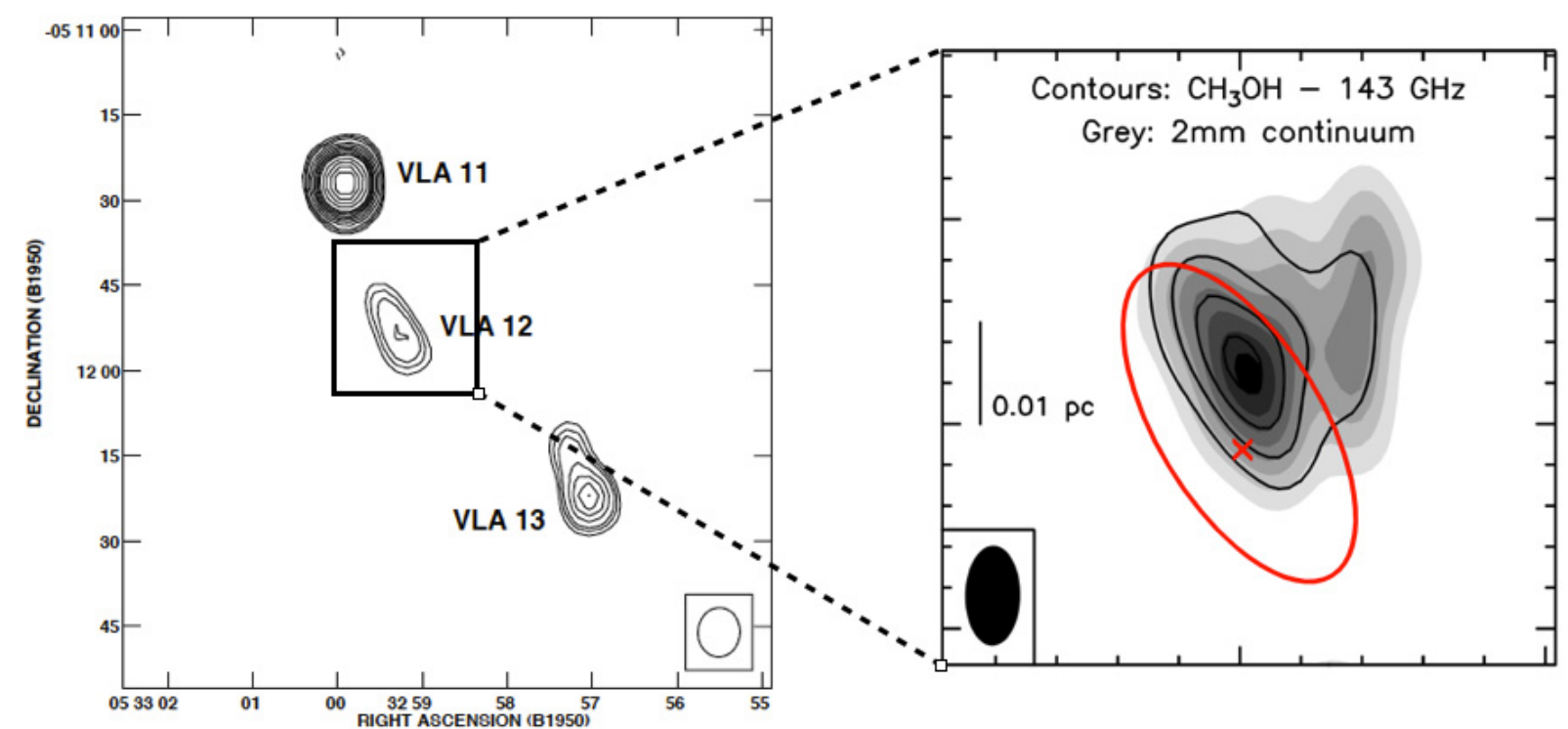

Fig. 10. Left panel: VLA map of the $3.6 \mathrm{~cm}$ continuum emission in the OMC-2 FIR 4 region and its surroundings (taken from Reipurth et al. 1999). The synthesised beam is shown at the bottom-right corner. The source labelled VLA 12 coincides with the position of OMC-2 FIR 4. Right panel: close-up view showing our PdBI continuum (grey scale) and $\mathrm{CH}_{3} \mathrm{OH}$ (black contours) maps. The synthesised beam is shown at the bottom-left corner. The red ellipse schematically represents the $4 \sigma$ contour of the VLA image (left panel). The red cross marks the position of the cm emission peak.

Reipurth et al. (1999) measured a flux of $0.64 \mathrm{mJy}$ at $3.6 \mathrm{~cm}$ towards OMC-2 FIR 4. In the scenario of an optically thin HII region, and assuming a single zero-age main sequence star is powering it, this implies a B3-B4 spectral type for the central star (using the tables of e.g. Panagia 1973; Thompson 1984; Martins et al. 2005). This translates into a luminosity between 700 and $1000 L_{\odot}$ and would indicate that this young star is the most massive source in the protocluster. It is also possible, given the elongated shape of the radio emission, that two (or more) individuals are responsible for the ionisation of their surroundings. Only higher angular resolution and sensitivity images of the continuum emission at different wavelengths will allow us to verify this possibility and find out whether the nature of the centimetre emission is an HII region, as proposed here, or a radio jet. 


\subsection{A final note on luminosities}

There is some confusion regarding the luminosity of OMC2 FIR 4 in the literature. While the first estimates by Mezger et al. (1990), and later by Crimier et al. (2009), report a value between $400 L_{\odot}$ and $1000 L_{\odot}$, a recent work by Adams et al. (2012) yields $50 L_{\odot}$. In what follows we try to understand the reason for this discrepancy.

The first point to mention is the complexity of the region. Indeed, the morphology and size of OMC-2 FIR 4 change when seen at different wavelengths. In the mid-IR it appears to be a compact source, while at wavelengths above $\sim 100 \mu \mathrm{m}$ its emission is more extended ( $30^{\prime \prime}$ in diameter) and peaks around $5^{\prime \prime}$ west of the mid-IR source. This said, we note that the luminosity estimates published by the different authors refer to different components. On the one hand, Adams et al. (2012) built the SED of the source by measuring the continuum fluxes, at IR and submm wavelengths, of the compact source they detect at $19 \mu \mathrm{m}$ and $37 \mu \mathrm{m}$ with SOFIA. In other words they adopted small aperture sizes, ranging between $7^{\prime \prime}$ and $13^{\prime \prime}$ and centred on the midIR peak. On the other hand, Crimier et al. (2009) and Mezger et al. (1990) took into larger areas into account, which are 30" and $50^{\prime \prime}$ in diameter, respectively, which include the whole envelope of OMC-2 FIR 4. In particular,

- Crimier et al. (2009) used far-IR data from ISO and IRAS, whose coarse angular resolution likely led to measured fluxes that were overestimated, owing to possible contamination from the nearby protostar FIR 3 , by at most a factor 2 , since at such wavelengths the dominant emission source is FIR 4.

- Adams et al. (2012) obtained Herschel-PACS maps of OMC2 FIR 4 from which they measured the fluxes at $70 \mu \mathrm{m}$ and $160 \mu \mathrm{m}$. They did so by applying small apertures of 9 .' 6 and 12 '. 8 , respectively, around the mid-IR peak coordinates, and a background annulus that actually covers part of the extended emission of the region. In addition, they carried out APEX observations at $350 \mu \mathrm{m}$ and $850 \mu \mathrm{m}$ that lacked the sensitivity needed to detect any emission. They therefore measured an upper limit of the flux considering only the emission within a beam size $\left(7.3^{\prime \prime}\right.$ and $19^{\prime \prime}$, respectively), again around the mid-IR coordinates, and therefore offset by $\sim 5^{\prime \prime}$ from the actual sub-mm peak position. As a result, their reported sub-mm fluxes are respectively ten and three times lower than those detected and measured by Crimier et al. (2009) with the CSO and JCMT telescopes. Considering these points, the authors may have underestimated the flux of OMC-2 FIR 4 by a factor of up to ten.

In short, it appears that the discrepancy in luminosity estimates found in the literature is caused by the fact that different authors measure different components of OMC-2 FIR 4, with Adams et al. (2012) concentrating on the compact mid-IR source and Crimier et al. (2009) on the entire envelope, which is several times larger. Table 9 summarises the different values for the luminosity, mass, and size of OMC-2 FIR 4 reported in the literature. Based on the discussion above, the actual value depends on how one defines OMC-2 FIR 4 and its boundaries. If our interpretation of an HII region is correct (see Sect. 4.3), the luminosity of the whole protocluster could be around several hundred solar luminosities.
Table 9. Mass and luminosity estimates for OMC-2 FIR $4^{a}$.

\begin{tabular}{cccc}
\hline \hline $\begin{array}{c}L \\
\left(L_{\odot}\right)\end{array}$ & $\begin{array}{c}M \\
\left(M_{\odot}\right)\end{array}$ & $\begin{array}{c}\text { Size } \\
\left({ }^{\prime \prime}\right)\end{array}$ & Ref. \\
\hline 350 & 34 & 50 & Mezger et al. (1990) \\
920 & 30 & 30 & Crimier et al. (2009) \\
50 & 10 & 8 & Adams et al. (2012) \\
\hline
\end{tabular}

Notes. ${ }^{(a)}$ Values re-scaled to a distance of 420 pc.

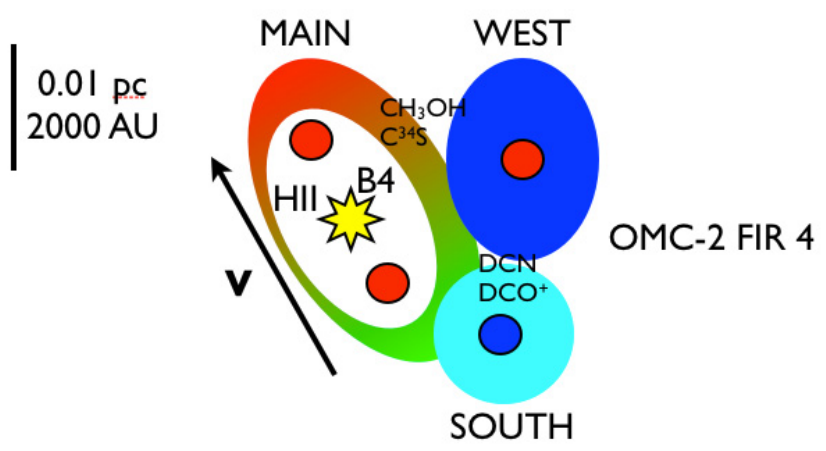

Fig. 11. Schematic cartoon of OMC-2 FIR 4 and the nearby OMC2 FIR 3, summarising all the physical elements in the region that we propose according to the data presented in this work (see Sect. 4). The white ellipse inside the main source depicts the ionised region powered by a B 4 young star. The colours of the main, west, and south sources represent their systemic velocities with respect to the nominal value for OMC-2 FIR $4\left(V_{\mathrm{LSR}}=11.4 \mathrm{~km} \mathrm{~s}^{-1}\right)$, marked in green. The small red and blue circles within the three sources represent the possibility that they harbour smaller unresolved molecular condensations, with the colour blue denoting a colder temperature than red.

\section{Conclusions}

We have presented new interferometric observations carried out towards the intermediate-mass protocluster OMC-2 FIR 4 in the Orion A complex. These include PdBI maps of the continuum emission at $143.4 \mathrm{GHz}(2 \mathrm{~mm})$ and the $\mathrm{CH}_{3} \mathrm{OH}(3,1$, $+0-2,1,+0), \mathrm{C}^{34} \mathrm{~S}(3-2), \mathrm{DCN}(2-1)$, and $\mathrm{DCO}^{+}(2-1)$ lines, as well as VLA maps of the $\mathrm{NH}_{3}(1,1)$ and $(2,2)$ inversion transitions. Despite the coarse channel spacing of the observations $\left(0.6 \mathrm{~km} \mathrm{~s}^{-1}\right.$ for the VLA spectra and $4 \mathrm{~km} \mathrm{~s}^{-1}$ for the PdBI images), we were able to extract important information about the morphology and kinematics of the region. Figure 11 shows a cartoon of the OMC-2 FIR 4/FIR 3 region, summarising all the elements present according to our interpretation of the data presented in this work. Our findings are summarised as follows.

1. Our 2-mm continuum maps reveal two main components and hints of secondary cores or clumps surrounding it. The existence of the 11 cores discovered by S08 could not be confirmed by our observations, despite our higher angular resolution.

2. Each PdBI line traces the region differently. By comparing their emission, we were able to distinguish three separate sources of one or several solar masses each, which we have named main, west, and south. The main and west ones are warmer. The south source displays a stronger line emission in $\mathrm{DCO}^{+}(2-1)$, suggesting a colder temperature and therefore an earlier evolutionary state or lower mass core. Alternatively, it may be associatied with part of the colder envelope of OMC-2 FIR 4. As for the ammonia maps, their emission is more extended and essentially single-peaked, 
and therefore traces the envelope of the protocluster. An LVG modelling of the $\mathrm{CH}_{3} \mathrm{OH}$ lines we observed allowed us to conclude that the whole protocluster is very dense, with $n \gtrsim 2 \times 10^{6} \mathrm{~cm}^{-3}$, in agreement with previous theoretical predictions on the structure of the envelope of OMC-2 FIR 4.

3 . The main source as seen by the $2-\mathrm{mm}$ continuum and $\mathrm{CH}_{3} \mathrm{OH}$ emission coincides, both in position (within the pointing errors) and in shape, with the radio-continuum emission observed at $3.6 \mathrm{~cm}$ with the VLA (Reipurth et al. 1999). In the absence of clear interferometric evidence of a molecular outflow driven by FIR 4, we interpreted this as an HII region powered either by a B3-B4 type young star or by two or more lower-mass stellar objects. This region is likely surrounded by a shell of dust and molecular gas (seen by the millimetre continuum and $\mathrm{CH}_{3} \mathrm{OH}$ maps). This scenario still needs to be verified by higher angular resolution and multiwavelength observations.

Thanks to its relative proximity to the Sun, OMC-2 FIR 4 is an ideal laboratory to explore intermediate- and low-mass star formation in the context of a whole protocluster. The evidence of core multiplicity in this region, coupled with the insufficient spatial resolution of our observations to clearly separate them, highlights the need of higher spectral and angular resolution imaging to disentangle the different components and extract more information about the kinematics and the excitation conditions in each of them. This information will be crucial for interpreting the Herschel-HIFI data obtained towards this source within the CHESS key programme.

Acknowledgements. A.L.S. and C.C. acknowledge funding from the CNES (Centre National d'Études Spatiales) and from the Agence Nationale pour la Recherche (ANR), France (project FORCOMS, contracts ANR-08-BLAN-022). M.K. gratefully acknowledges funding from an NWO grant, NOVA, Leids Kerkhoven-Bosscha Fonds, and the COST Action on Astrochemistry. C.D. acknowledges funding from Leids Kerkhoven-Bosscha Fonds. A.F. has been partially supported within the programme CONSOLIDER INGENIO 2010, under grant CSD2009-00038 "Molecular Astrophysics: The Herschel andALMA Era ASTROMOL". We are grateful to the anonymous referee and to C.M. Walmsley, whose suggestions contributed to improve the quality of the present paper.

\section{References}

Adams, J. D., Herter, T. L., Osorio, M., et al. 2012, ApJ, 749, L24

Beuther, H., Churchwell, E. B., McKee, C. F., \& Tan, J. C. 2007, in Protostars and Planets V, eds. B. Reipurth, D. Jewitt, \& K. Keil, 165

Bottinelli, S. 2006, Ph.D. Thesis, University of Hawaii at Manoa

Busquet, G., Palau, A., Estalella, R., et al. 2009, A\&A, 506, 1183
Ceccarelli, C., Maret, S., Tielens, A. G. G. M., Castets, A., \& Caux, E. 2003, A\&A, 410, 587

Ceccarelli, C., Caselli, P., Herbst, E., Tielens, A. G. G. M., \& Caux, E. 2007, in Protostars and Planets V, eds. B. Reipurth, D. Jewitt, \& K. Keil, 47 Ceccarelli, C., Bacmann, A., Boogert, A., et al. 2010, A\&A, 521, L22 Cesaroni, R., \& Wilson, T. L. 1994, A\&A, 281, 209

Chini, R., Reipurth, B., Ward-Thompson, D., et al. 1997, ApJ, 474, L135

Crapsi, A., Caselli, P., Walmsley, C. M., et al. 2005, ApJ, 619, 379

Crimier. N., Ceccarelli, C., Lefloch, B., \& Faure, A. 2009, A\&A, 506, 1229

Crimier, N., Ceccarelli, C., Alonso-Albi, T., et al. 2010, A\&A, 516, A102

de Graauw, Th., Helmich, F. P., Phillips, T. G., et al. 2010, A\&A, 518, L6

Foster, J. B., Rosolowsky, E. W., Kauffmann, J., et al. 2009, ApJ, 696, 298

Fuente, A., Ceccarelli, C., Neri, R., et al. 2007, A\&A, 468, 37

Fuente, A., Castro-Carrizo, A., Alonso-Albi, T., et al. 2009, A\&A, 507, 1475

Hildebrand, R. H. 1983, QJRAS, 24, 267

Johnstone, D., \& Bally, J. 1999, ApJ, 510, L49

Johnstone, D., Boonman, A. M. S., \& van Dishoeck, E. F. 2003, A\&A, 412, 157

Jørgensen, J. K., Schöier, F. L., \& van Dishoeck, E. F. 2004, A\&A, 416, 603

Jørgensen, J. K., Johnstone, D., van Dishoeck, E. F., \& Doty, S. D. 2006, A\&A, 449, 609

Kama, M., Dominik, C., Maret, S., et al. 2010, A\&A, 521, A39

Kama, M., López-Sepulcre, A., Dominik, C., et al. 2013, A\&A, submitted, DOI: 10.1051/0004-6361/201219431

Kim, M. K., Hirota, T., \& Honma, M. 2008, PASJ, 60, 991

Li, D., Kauffmann, J., Zhang, Q., \& Chen, W. 2012, ApJ, 768, 5

Lis, D. C., Serabyn, E., \& Keene, J. 1998, ApJ, 509, 299

Liu, Q., Yang, J., Sun, Y., \& Xu, Y. 2011, RA\&A, 11, 67

Martins, F., Schaerer, D., \& Hillier, D. J. 2005, A\&A, 436, 1049

Menten, K. M., Reid, M. J., Forbrich, J., \& Brunthaler, A. 2007, A\&A, 474, 515

Mezger, P. G., Wink, J. E., \& Zylka, R. 1990, A\&A, 228, 95

Nielbock, M., Chini, R., \& Müller, S. A. H. 2003, A\&A, 408, 245

Olmi, L. Angles-Alcazar, D., Elia, D., et al. 2013, A\&A, 551, A111

Ossenkopf, V., \& Henning, T. 1994, A\&A, 291, 943

Palau, A., Sánchez-Monge, Á., Busquet, G., et al. 2010, A\&A, 510, A5

Palau, A., Fuente, A., Girart, J. M., et al. 2011, ApJ, 743, 32

Panagia, N. 1973, AJ, 78, 929

Pottage, J. T., Flower, D. R., \& Davis, S. L. 2004, MNRAS, 352, 39

Reipurth, B., Rodríguez, L. F., \& Chini, R. 1999, ApJ, 118, 983

Sánchez-Monge, Á., Palau, A., Estalella, R., et al. 2010, ApJ, 721, L107

Shimajiri, Y., Takahashi, S., Takakuwa, S., Saito, M., \& Kawabe, R. 2008, ApJ, 683,255

Shu, F. H., Adams, F. C., \& Lizano, S. 1987, Ann. Rev. A\&A, 25, 23

Takahashi, S., Saito, M., \& Ohashi, N. 2008, ApJ, 688, 344

Tatematsu, K., Umemoto, T., \& Kameya, O. 1993, ApJ, 404, 643

Tatematsu, K., Kandori, R., Umemoto, T., \& Sekimoto, Y. 2008, PASJ, 60, 407

Testi, L., Palla, F., \& Natta, A. 1999, A\&A, 342, 515

Thompson, R. I. 1984, ApJ, 283, 165

van Kempen, T. A., Longmore, S. N., Johnstone, D., \& Pillai, T. 2012, ApJ, 751, 137

Williams, J. P., Blitz, L., \& McKee, C. F. 2000, in Protostars and Plantes IV (Tucson: Univ. Arizona Press), 97

Wilner, D. J., \& Welch, W. J. 1994, ApJ, 427, 898

Wootten, A. 1995, Ap\&SS, 224, 43

Zinnecker, H., \& Yorke, H. W. 2007, ARA\&A, 45, 481 